\title{
The Ethics of Infectious Disease Control: \\ Lessons from the Ebola outbreak and an ethical framework.
}

\author{
By \\ Joshua McIvor
}

A thesis submitted to the Victoria University of Wellington in fulfilment of the requirements for the degree of Master of Arts in Philosophy.

Victoria University of Wellington.

February $29^{\text {th }}, 2016$. 
Joshua Mclvor, The Ethics of Infectious Disease Control.

\section{Abstract:}

Ebola virus disease (EVD) devastated its way into news headlines in 2014, destroying communities across three West African countries and costing the lives of over 11,000 people. The global health response was widely scrutinised and criticised, and though the outbreak is now over, there are still many lessons that can be learned from the 2014 EVD outbreak. This thesis will use the EVD outbreak in two ways. Firstly, I will use the EVD outbreak as a case study through which I will strive to address the ethical concerns for using experimental treatment during the outbreak, and I will address ethical concerns of the use of quarantine during the outbreak. Second, I will use the EVD outbreak as a launch pad to examine broader and more abstract ethical principles of the ethics of infectious disease control, such as the principles of reciprocity, transparency, proportionality, and the harm principle. This discussion will highlight how physical, biological features of a disease very much impact the application of the above principles when it comes to controlling the disease in an ethical manner. Finally, from this observation, I have created a 'disease taxonomy' that categorises infectious diseases based upon, what I argue, are the most ethically relevant biological features of infectious diseases. The taxonomy can aid in preparing for, understanding, and responding to the most pertinent ethical issues that surround various infectious diseases. The thesis should leave the reader with not only a greater understanding of some of the ethical issues raised by the 2014 EVD outbreak, but also a solid framework to utilise in discussing the most pertinent ethical issues of any future outbreak of any infectious disease. 
Joshua Mclvor, The Ethics of Infectious Disease Control.

\section{Contents:}

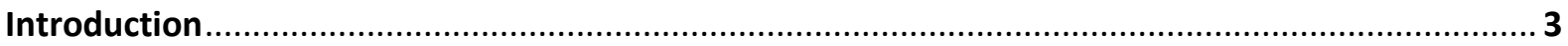

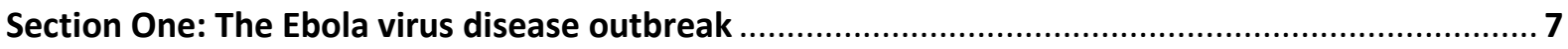

1.1 What is Ebola Virus Disease and why was the outbreak so bad? .............................................. 7

1.2 An Ethical Issue: the use of experimental drugs during the outbreak........................................ 12

1.2.1 Why was there no widespread treatment for EVD already? ......................................... 12

1.2.2 Was the use of experimental drugs during the EVD outbreak ethical?......................... 13

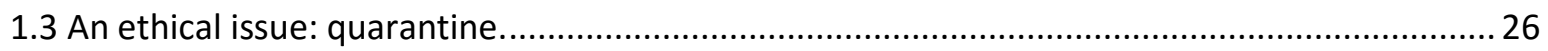

1.4 Conclusions: Lessons from the EVD outbreak and moving forward .......................................... 29

Section Two: Do the ethics of infectious disease control vary depending on the disease? .............. 32

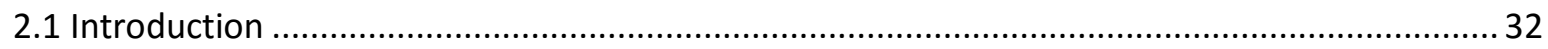

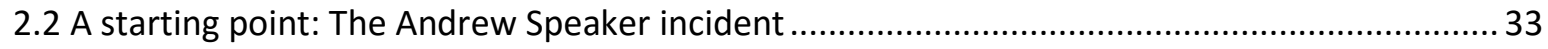

2.3 The foundation of infectious disease control: the principle of reciprocity …............................. 35

2.4 Broadening principles: transparency, proportionality, and harm ............................................ 40

2.5 Do the ethics of infectious disease control vary depending on the disease? .............................. 44

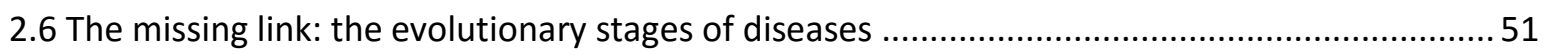

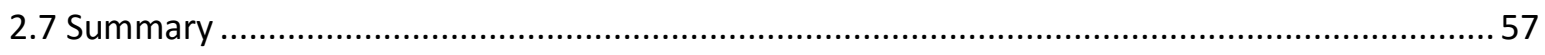

Section Three: A Disease Taxonomy, Ethical and Policy Implications ............................................. 59

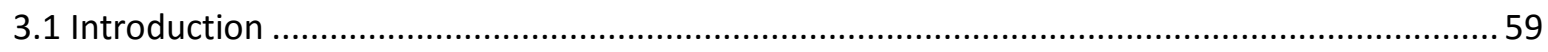

3.2 Current approaches to infectious disease control in New Zealand .........................................60

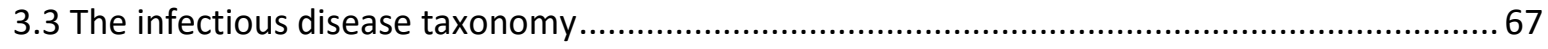

3.4 An ethical framework for the infectious disease taxonomy .................................................. 72

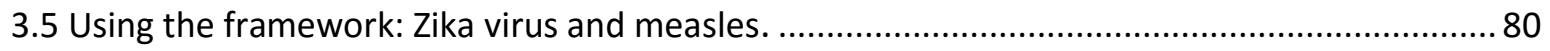

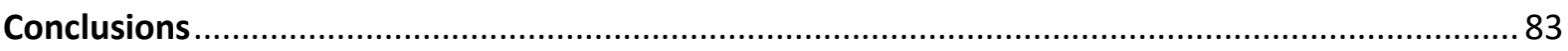

References

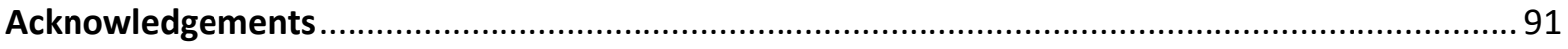

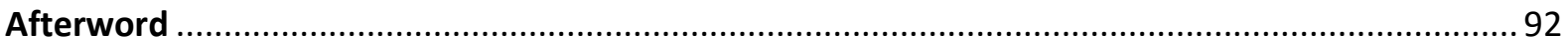


Joshua Mclvor, The Ethics of Infectious Disease Control.

\section{Introduction.}

Plague, Smallpox, Yellow Fever, Malaria, Influenza, Polio, Tuberculosis (TB), Human Immunodeficiency Virus (HIV), Ebola, and Severe Acute Respiratory Syndrome (SARS) are some of the diseases that have devastated humanity over the last several centuries and in some cases longer. Antibiotic resistance, the 2014 Ebola Virus Disease (EVD) outbreak in West Africa, the current outbreak of Zika virus in Brazil, and seasonal influenza are some of the frequently reported reminders of the perilous relationship humans have with the microbes of this world. As the global population increases, crowding becomes more common, and international travel more frequent, the risk pathogens pose to the global population rises. The relative isolation much of the globe experienced only a few centuries ago is diminishing and as such our ability to control the spread of infectious diseases is being challenged. The need for infectious disease control policy that is responsive, effective, and adaptive, is apparent. However, ethical issues concerning infectious disease control mustn't be ignored and must be incorporated into any reasonable policy. Weighing up individual rights against the need to protect the greater society is a difficult task; leaning too far in either direction is to neglect the proper ethical analysis of the problem at hand. The purpose of this paper is to create an ethical framework to be used during infectious disease control. Though I will criticise aspects of current policy, the framework is not necessarily intended to be used instead of current policy, but in addition to current policy. I hope to offer a framework that allows for the flexibility needed when dealing with both known and novel or little-known - pathogens, whilst allowing for the incorporation of well-established policy with regards to well-known pathogens. I hope to achieve this whilst maintaining a high degree of ethical consideration. The major questions I will address are:

1) When, if ever, is it okay to use experimental treatment?

2) When is quarantine/isolation ethically justified ? $^{1}$

3) What are the key ethical principles to adhere to when it comes to infectious disease control?

4) Does our application of ethical principles vary between diseases?

\footnotetext{
${ }^{1}$ Questions one and two will be the key focus when examining the 2014 EVD outbreak.
} 
Joshua Mclvor, The Ethics of Infectious Disease Control.

However, in order to have an idea of what good ethical policy may look like regarding infectious disease control, we must have a clear understanding of some of the established key points of discussion on the topic. We could dive straight into some of the broader or abstract discussions on the issue, yet I think it best to begin with a specific case-study and some of the ethical questions it raises. I do not want this paper to be an abstruse piece of writing, understood only by those who already have some knowledge of the issues in the ethics of infectious disease control. Rather, I want this paper to be widely comprehensible and accessible to those who may not be familiar with ethics or philosophy. As such, I want to minimise assumptions made and take a bottom-up approach, which leads the reader to the conclusions in a logical and coherent manner. Therefore, it seems natural to begin the discussion with a specific case study, that many should be familiar with, in order to provide some context before moving on to some broader points. So, to begin, I will examine the 2014-2015 West Africa EVD outbreak and some of the ethical issues the crisis raised.

I will be utilising the EVD outbreak in two major ways. Firstly, I will use the EVD outbreak as a case study through which I will strive to address the ethical concerns for using experimental treatment during the outbreak, and I will address the ethical concerns of the use of quarantine during the outbreak. Second, I will use the EVD outbreak as a launch pad to examine broader and more abstract ethical principles of the ethics of infectious disease control, such as the principles of reciprocity, transparency, proportionality, and the harm principle.

My hope is that the case study will provide both a real world example of how the ethics of infectious disease control is far from black and white, as well as provide some empirical information as to how EVD works and why it was so difficult to control during this outbreak. A key point of discussion will be whether the use of experimental drugs on patients and staff is ethical in this context. An important question to reflect upon throughout this first section will be: what could have been done better? The purpose of this question is not to impart blame, but instead learn from shared experiences. The outbreak was and is a global problem. Thus, it is not only the duty of the countries affected to analyse the response taken to the crisis and strive to do better the next time a relatively novel outbreak occurs, but all countries must consider this. Once we understand the EVD outbreak and perhaps what could have been done better, we need to ask ourselves the question: do the lessons 
Joshua Mclvor, The Ethics of Infectious Disease Control.

we've learnt from the EVD outbreak apply to all diseases? That is to say, do/should the differences between infectious diseases affect what it means to have an ethical response to any given disease outbreak? Do the physical facts and features of diseases change what courses of action are ethically appropriate when dealing with said diseases? In section two of this paper I will examine this question in detail. I will analyse a number of different infectious diseases, as well as some of the general principles of infectious disease control examined by other philosophers and bioethicists. By reviewing papers that break down the ethics of infectious disease control to its most basic level, such as John Harris and Soren Holm's "Duty not to infect others" ${ }^{2}$, I hope to provide a picture of exactly how and why we may feel differently about what is considered ethical when dealing with, say, SARS as opposed to rhinovirus (the common cold).

This section will utilise four major ethical principles:

1) The principle of reciprocity, as understood and outlined by Harris and Holm.

2) The principle of harm.

3) The principle of proportionality.

4) The principle of transparency. ${ }^{3}$

These principles will form the foundation for understanding appropriate ethical action across all infectious diseases. Yet, our answers to the questions the principles pose, will change based upon physical facts of the diseases.

Furthermore, I would like to emphasise that whilst our ethical outlook may change depending on the physical differences between diseases, this is not a case of us changing our ethics or ethical theory. I do not wish to encourage forsaking ethical standards in the face of adversity and simply cave to pragmatic constraints. Rather, I would like this flexibility to be viewed as an application of a singular ethical framework that not only allows practical concerns to affect our ethical outlook, but suggests that practical concerns are very much a part of the ethical theory itself. This will become clear later.

After showing that appropriate ethical actions may differ between infectious diseases, I would like to formally lay out a type of disease taxonomy that categorises

\footnotetext{
${ }^{2}$ Harris \& Holm, Is There A Moral Obligation Not To Infect Others?

${ }^{3}$ Principles two, three, and four I will be taking from: Upshur, The Ethics of Quarantine.
} 
Joshua Mclvor, The Ethics of Infectious Disease Control.

infectious diseases based on what I consider to be their most ethically relevant biological features. From here, it is possible to make ethical claims that outline the appropriate ethical actions and considerations for each of the disease taxonomy categories. This section of the paper will be the most applied, in that it will attempt to examine actual official New Zealand policy. Despite my optimism as to the benefits of my framework, it will undoubtedly be open to much criticism. Ultimately, I am both more and less constrained than actual policy makers. In one respect, being a philosopher, I can afford to be a lot more idealistic when it comes to the ethical considerations I make when suggesting real world policy amendments. Although I will try to take into account as many practical constraints as possible, I will inevitably fall short of the kind of knowledge real policy makers have in their area of expertise. In another respect, I am more constrained in that I am not in fact a policy maker and thus lack many of the resources available to policy makers. This is not to excuse my culpability as the writer of this paper. It is more to say that my suggestions, guided by ethical theory, are still ultimately just that: suggestions. I would be very much open to constructive criticism that suggests alterations that would aid in making my suggestions more viable or even uphold a greater ethical standard. My suggestions stand insofar as the context of this thesis allows: if disagreement with current policy arises it will be adequately criticised, yet I will acknowledge my limitations and accept that a defence of such policy may very well stand up to my suggestions if the discussion were to be pursued. I will discuss this matter further in the conclusion of this paper. 
Joshua Mclvor, The Ethics of Infectious Disease Control.

\section{Section One: The Ebola virus disease outbreak.}

\subsection{What is Ebola Virus Disease and why was the outbreak so bad?}

Ebola, more technically Ebola Virus Disease (EVD), is a haemorrhagic disease that is severe and often fatal. It has a roughly $50 \%$ mortality rate, but case fatality rates have ranged from $20 \%$ to $90 \%$ in the past. It originates in animals, the leading candidate of which is fruit bats and was first seen in 1976 in two simultaneous outbreaks: one in Sudan and the other in the Democratic Republic of Congo (DRC). The second outbreak occurred near the Ebola river, from which the virus gets its name. The disease initially causes fever, fatigue, muscle pain, headache, and a sore throat. Subsequently, the patient will suffer vomiting, diarrhoea, rash, symptoms of impaired kidney and liver function, and in some cases, both internal and external bleeding. Treatment for EVD is limited; however there are some promising options. ${ }^{4}$ The World Health Organisation (WHO) elaborates:

\footnotetext{
"Supportive care rehydration with oral or intravenous fluids and treatment of specific symptoms, improves survival. There is as yet no proven treatment available for EVD. However, a range of potential treatments including blood products, immune therapies and drug therapies are currently being evaluated. No licensed vaccines are available yet, but 2 potential vaccines are undergoing human safety testing." ${ }^{5}$
}

Despite the severity of EVD, it is definitely possible to control the disease when adhering to strict hygiene practices. Before explaining why, I will need to outline some common terminology. My understanding is that there is some inconsistency with the utilisation of the following terms, so it is important that the terms are clearly defined for the purposes of this thesis. I will be using Kenrad E. Nelson's terminology in this paper: ${ }^{6}$

- Virulence: Fatality of the disease: diseases that are highly virulent are more severe and have a higher fatality rate.

- Infectivity: The number of infectious particles that are required to establish an infection in the host (i.e. the host is considered infected with the disease and is a carrier). This can be measured by determining the number of individuals who become infected with the disease after exposure to the disease.

\footnotetext{
${ }^{4}$ WHO factsheet, Ebola virus disease.

5 Ibid, pg. 1.

${ }^{6}$ Nelson, Infectious Disease Epidemiology $-3^{\text {rd }}$ Ed, pg. 36-37.
} 
Joshua Mclvor, The Ethics of Infectious Disease Control.

- Pathogenicity: A measure of the microbial agent's ability to induce disease in the host, after infection. Hosts infected with a disease of low pathogenicity, such as polio, may not present actual symptoms of the infection.

- Immunogenicity: The ability of the disease to produce an immune response in the host that will ultimately provide protection against reinfection. Diseases that are strongly immunogenic are ideal candidates for vaccination, as exposure to the disease can lead to lifelong protection from reinfection.

- Inapparent Infection: Diseases that have low pathogenicity may have a high inapparent infection rate. Infection of a host can be determined by laboratory testing, yet a host my present no symptoms. Diseases with high inapparent infection rates are difficult to control, as it is very difficult to determine which individuals are infected with the disease.

This terminology will become very important later in the paper. As I suggested in the introduction, biological features of infectious diseases are important when it comes to the ethics of infectious disease control. The four principles I stated in the introduction will require us to understand the biological features of infectious diseases if we are to adhere to the principles when making ethical considerations for the control of such diseases. Therefore, it is important that the reader understands the above terminology in order to properly engage with the paper later on and they may wish to turn back to this page when reading through the rest of the paper.

Now that we have some clearly defined terminology, we can examine exactly why preventing the spread of EVD should be theoretically easier than, say, influenza. EVD has a high virulence, infectivity, and pathogenicity, which is why it is such a devastating disease. Yet there are two saving graces when it comes to preventing the spread of Ebola. Firstly, a susceptible host must come into contact with bodily fluids of an infected individual (or animal) in order to contract the disease. Though this may not sound like much of a saving grace, as the bodily fluids of an individual can contaminate a surface or material that can subsequently contaminate another human, when paired with the second saving grace, practitioners are left with a disease that, though certainly fierce, can be beaten by relatively straightforward protocols. The second saving grace is that individuals infected with EVD are only contagious once they are symptomatic. This means the spread of the disease, from one 
Joshua Mclvor, The Ethics of Infectious Disease Control.

person to another, won't occur when the initial host is unaware that they are infected. This combination provides some key benefits when it comes to managing the spread of the disease. For one, in an uncontrolled situation, such as a contagious individual being present in a crowd, there are likely to be fewer individuals infected than if the disease were airborne (of course infectivity and pathogenicity will play a big role here, but I mean to compare as if the EVD virus itself was airborne, with its same properties). Second, because an individual needs to be symptomatic in order to be contagious, even untrained professionals can recognise when an individual needs to be isolated (if they understand the connection between contagiousness and symptoms). Third, health care professionals can utilise physical barriers to protect themselves and others from infected patients. This can range from physical isolation in a room, to wearing protective clothing. Some of these protection techniques may not work as well, if at all, for certain airborne diseases. With the right protection and hygiene practices, spread of the disease can be stemmed dramatically, as did eventually occur with the 2014 EVD outbreak.

So, what made the 2014 EVD outbreak so bad? Essentially: a perfect storm of negative factors. A preliminary or background factor, is that EVD is what Nathan Wolfe, Claire Panosian Dunavan, and Jared Diamond refer to as a "stage three" disease. ${ }^{7}$ I will elaborate more on Wolfe et al. in the second section of this paper, but for now it is important to understand that a stage three disease, is one that has crossed from animals to humans, but will usually only undergo a few cycles of secondary transmission (human to human) before dying out. This means, due to the nature of the disease, stage three diseases tend to flare up in a population, before dying out as a result of the pathogen being unable to sustain itself for an extended period time outside of its reservoir host. The result is that the disease is very hard to study outside of outbreaks, especially if the reservoir host is unknown. Though the fruit bat is suspected as the reservoir host for EVD, this has not been definitively proven, and studying the disease is difficult. ${ }^{8}$ In addition, the economic incentive for large pharmaceutical companies to create treatments for diseases that only flare up infrequently is less than that of diseases that persist in humans for extended periods of time, such as influenza. The result of these factors is a population (local and international)

\footnotetext{
${ }^{7}$ Wolfe, Dunavan, \& Diamond, Origins of major human infectious diseases.

${ }^{8}$ For an account of the difficulties of studying the disease, as well as a detailed history of the disease I recommend reading "Ebola" by David Quammen, listed in the references section.
} 
Joshua Mclvor, The Ethics of Infectious Disease Control.

that was wildly underprepared for any EVD outbreak, let alone one on the scale of the 2014 outbreak.

The fact of the matter is the international community and health sector was desperately underprepared for an EVD outbreak anywhere on earth, and what made the situation worse was that the outbreak occurred in very poor region of the planet. There are many papers explaining why the 2014 EVD outbreak was so bad and what we can learn from the situation to prevent a repeat of the incident in the future. ${ }^{9}$ I will summarise some of the key issues that were encountered in this particular outbreak. Firstly, as I said earlier, the outbreak occurred in desperately poor countries with broken healthcare systems. ${ }^{10}$ In the affected countries, the healthcare systems are underfunded and lack the resources necessary to combat an outbreak. With a lack of protective equipment, hospital staffs were sometimes reluctant to go to work, out of a justified fear of contracting the disease themselves. Patients were reluctant to seek medical assistance at the hospitals for fear that if they did not already have EVD, they would certainly catch it if they went to hospital. This also meant that those who were in a position where they could potential help transport patients to hospital were also reluctant to assist, for fear of catching the disease. The healthcare systems were stretched to capacity and rendered largely ineffective in containing the disease, so there was minimal public trust in the healthcare system. ${ }^{11}$

Secondly, the deployment of military personnel to attempt to contain the disease frightened much of the population. Cordons were created to restrict movement and the spread of the disease. ${ }^{12}$ However, it often left communities without the basic resources necessary to continue life and prevented those that were sick from accessing medical care. By and large, entire communities became robbed of their basic human rights, as governments scrambled to prevent the spread of the disease. This had the effect of further reducing public trust in the government's ability to protect them from the disease. Cases like this are excellent examples of a catastrophic failure to uphold the moral principles I

\footnotetext{
${ }^{9}$ Donovan, Ebola, epidemics, and ethics - what we have learned; Gostin, West Africa's Ebola Epidemic is Out of Control, but Never Had to Happen; and Omonzejele, Ethical Challenges Posed by the Ebola Virus Epidemic in West Africa, are three excellent papers that cover some of the ethical issues the 2014 EVD outbreak posed, as well as a critical assessment of the global response.

${ }^{10}$ Gostin, West Africa's Ebola Epidemic is Out of Control, but Never Had to Happen, pg. 2.

11 Ibid.

12 Ibid, pg. 3.
} 
Joshua Mclvor, The Ethics of Infectious Disease Control.

mentioned earlier in the paper. Had these principles been upheld, the result would much better than what I have described above.

Finally, there was a widespread lack of public education regarding the disease and how to protect from it. There was a prevalence of misconceptions about EVD and accurate news reporting was curtailed by governments. ${ }^{13}$ As a result, traditional cultural practices that aided in the spread of Ebola, were not strongly educated against. Local burial practices that involved close contact with the bodies of the deceased were widespread and largely contributed to the spread of the disease, often ravaging entire families. Burial practices included hand washing of the bodies and it should be noted that funerals are traditionally a large affair, with many friends and family of the deceased coming into close contact with the body. Needless to say, these practices did not help contain the spread of the disease; where, in other circumstances, a deceased host would usually mark the end of that particular vector of the disease. Another local practice - eating bush meat - proved risky when it came to the spread of EVD. Though often illegal, the killing and selling of bush meat is wide spread in sub-Saharan Africa. Unsurprisingly, when a disease is largely animal borne, this leads to an increase in incidences of the disease during an outbreak. This was the case with EVD. It is difficult to educate and encourage against the eating of bush meat as the practice is, for many poor Africans, both traditional and a source of valuable protein. ${ }^{14}$ Some even go as far as to claim the EVD outbreak isn't even real and that healthcare professionals themselves were killing patients in order to receive western funding. ${ }^{15}$ Clearly, greater public education was needed to prevent the spread of EVD as well as restore trust in the health care systems.

So, a whole host of factors created the perfect storm, which led to the 2014 West Africa EVD outbreak being the worst EVD outbreak in history. The difficulties of employing a largely unprepared global health care community against a disease we did not know much about, in one of the poorest regions one earth, where much of the population is uneducated and the healthcare systems are broken, led to the deaths of over 11,000 individuals. But what can we learn from the outbreak? To answer this question, we will need

\footnotetext{
${ }^{13}$ Gostin, West Africa's Ebola Epidemic is Out of Control, but Never Had to Happen, pg. 2

${ }^{14}$ Ibid.

${ }^{15}$ Donovan, Ebola, epidemics, and ethics - what we have learned, pg. 2.
} 
Joshua Mclvor, The Ethics of Infectious Disease Control.

to examine some of the major ethical quandaries that arose from the outbreak. First and most significantly I will look at the use of experimental treatment during the outbreak. Then, I will examine the use of quarantine during the outbreak. My hope is that by examining these issues, the reader can grasp some of the major ethical issues and challenges the 2014 EVD outbreak posed and thus get an idea as to the kind of hurdles that need to be overcome when dealing with infectious disease control more generally.

\subsection{An Ethical Issue: the use of experimental drugs during the outbreak.}

In August of 2014, when the EVD outbreak in West Africa was close to its peak, two American volunteers, Kent Brantly and Nancy Writebol contracted EVD while working in Liberia. They were treated with an experimental drug called Zmapp, which was a combination of three monoclonal antibodies that were never before administered to humans. ${ }^{16}$ The condition of Brantly and Writebol reportedly improved after receiving the drug. ${ }^{17}$ Though the survival of Brantly and Writebol, once receiving Zmapp, understandably brought hope to many, the survival of only two patients is hardly enough to establish the validity of the treatment, as a sample group of only two individuals is not a statistically significant group. Furthermore, preclinical and clinical trials are needed to determine the safety and efficacy of a new drug. ${ }^{18}$ Nevertheless, the whole ordeal generated much controversy in the media and raised a whole host of ethical questions surrounding the use of experimental treatment during an outbreak. I will attempt to break down some of these questions and offer answers that may indicate how we can collectively deal with future infectious disease outbreaks.

\subsubsection{Why was there no widespread treatment for EVD already?}

A common question that arises when the issue of experimental EVD treatment is raised is either "why was there was no vaccine already?" or "why was there so little of the only (potentially) effective treatment available (i.e. Zmapp)?" This is an understandable question and we do, after all, live in a world where many communicable diseases have an effective treatment, vaccine, or some kind of effective prevention. Additionally, we have known about EVD and its lethality since the late 70's. One of the reasons, rather sadly (though

\footnotetext{
${ }^{16}$ Gupta and Dellorto, Experimental drug likely saved Ebola patients.

${ }^{17}$ Goodman, Studying 'Secret Serums' - Toward Safe, Effective Ebola Treatments, pg. 1087.

${ }^{18}$ More on this: West's Encyclopaedia of American Law, Food and Drug Administration.
} 
Joshua Mclvor, The Ethics of Infectious Disease Control.

perhaps not unjustly), seems to be that there was little motivation for drug companies to research EVD treatments due to the fact that until 2014, outbreaks only affected dozens or at the most a few hundred individuals: there simply weren't enough people affected by the disease. ${ }^{19}$ Furthermore, the disease was confined to sub-Saharan Africa, one of the poorest regions on earth. There was simply little money to be made from the development of an effective treatment for EVD. Yet, there are also practical reasons it has been difficult to develop an effective treatment for EVD. Wolfe et al. categorise EVD as a stage 3 disease, meaning it can spread from animals to humans (primary transmission), and from humans to humans (secondary transmission), but normally only undergoes a few cycles of secondary transmission. Outbreaks, therefore, tend to burn themselves out. ${ }^{20}$ As said, up until the 2014 outbreak, EVD only flared up in small communities and for relatively short amounts of time. So, even researchers who have the motivation to develop an effective treatment for EVD will struggle when it comes to studying the disease. It would undoubtedly involve trekking into the depths of the African interior, through treacherous and hostile terrain. ${ }^{21}$ Furthermore, there would be limited opportunities to study the disease in humans, compared to the ample supply of humans infected with influenza every year. All in all, this may seem a pretty weak defence of why we don't (or didn't prior to the 2014 outbreak) have an effective treatment for EVD. We could examine the question of what moral obligations we have as a global community to prepare for diseases such as EVD, but that will not be in the scope of this thesis. ${ }^{22}$ For now, the above is by way of an explanation as to why no effective treatment was available for EVD prior to the 2014 outbreak.

\subsubsection{Was the use of experimental drugs during the EVD outbreak ethical?}

Despite there not being an effective treatment in place during the 2014 EVD outbreak, there had been research into EVD drugs and vaccines for the previous decade or so. The problem at the time was while "Some of these have shown promising results in the laboratory and in animal models... they have not yet been evaluated for safety and efficacy

\footnotetext{
${ }^{19}$ Donovan, Ebola, epidemics, and ethics - what have we learned, pg. 4.

${ }^{20}$ Wolfe, Dunavan, \& Diamond, Origins of major human infectious diseases, pg. 280.

${ }^{21}$ Quammen, Ebola: The Natural and Human History of a Deadly Virus.

22 If the reader is interested in one suggestion of how to be better prepared for future zoonotic-origin outbreaks, the following is an interesting proposal: Wolfe, Dunavan, \& Diamond, Origins of major human infectious diseases, pg. 283.
} 
Joshua Mclvor, The Ethics of Infectious Disease Control.

in humans." ${ }^{23}$ This put health care professionals and the WHO in a very difficult spot. As the WHO pointed out "The large number of people affected by the present outbreak in West Africa and the high case-fatality rate have prompted calls to accelerate the evaluation and development of these investigational medical interventions and to use them to try to save the lives of patients and curb the epidemic." ${ }^{24}$ Yet, despite public pressure, medical professionals must always ensure they act in a safe and ethical manner, and the matter of treating sick individuals with a treatment that has not yet been proven safe for humans, is a very risky business, due to the lack of clinical trials. It is an understandable urge for the general public to take an "anything is better than nothing" approach to experimental treatment, when we see thousands of individual's dying from a horrendous disease. That said, we cannot allow this very human drive to help others, cloud our judgement and we must remember the regulatory and safety framework in place when it comes to drug development, is there for a reason. So, the WHO decided to convene an emergency panel, inviting top bioethicists, scientists, medical professionals, and researchers from around the world, to discuss the ethics of using unregistered treatments during the 2014 EVD outbreak. ${ }^{25}$ The panel was held on the $11^{\text {th }}$ of August 2014 , five days after the American doctors, mentioned above, were treated with Zmapp. The report produced to outline the findings of the panel, was utilised for further academic discussion and critique of the WHO's findings. I will utilise the report, as well as supporting academic criticisms, to offer my own analysis of the ethics of using experimental treatment during infectious disease outbreaks.

The WHO panel were tasked with offering insight into five questions:

"1. Is it ethical to use unregistered interventions that have shown promising results in the laboratory and in animal models but have unknown adverse effects in humans for possible treatment of people who are infected? If yes, what criteria and conditions must be satisfied before they can be used?

2. Is it ethical to use unregistered interventions that have shown promising results in the laboratory and in animal models but have unknown adverse effects in humans for prophylaxis in people who are exposed but who show no signs of disease (i.e. post-exposure prophylaxis)? If yes, what criteria and conditions must be satisfied before they can be used?

\footnotetext{
${ }^{23}$ WHO, Ethical considerations for use of unregistered interventions for Ebola virus disease, pg. 1.

24 Ibid.
} 
Joshua Mclvor, The Ethics of Infectious Disease Control.

3. Is it ethical to use unregistered interventions that have shown promising results in the laboratory and in animal models but have unknown adverse effects in humans for prophylaxis in people who may be exposed (i.e. pre-exposure prophylaxis)? If yes, what criteria and conditions must be satisfied before they can be used?

4. If it is ethical to use unregistered interventions that have shown promising results in the laboratory and in animal models under the circumstances described above, what criteria should guide the choice of intervention?

5. If it is ethical to use unregistered interventions that have shown promising results in the laboratory and in animal models under the above circumstances, who should receive priority for treatment or prevention?"26

These issues boil down to a few major questions that need to be answered not only for the EVD outbreak, but also for potential future outbreaks of any infectious disease:

1) Is it ever ethical, during an outbreak, to use experimental treatments that have not been proven safe on humans...

a. That are infected?

b. That risk becoming infected?

2) What conditions must be met in order for such treatment to be given?

3) How do we prioritise treatment if the resources are limited?

In answer to the first question, the WHO panel said 'yes'. They concluded that it is ethical to use experimental treatment during the EVD outbreak, and there is even an ethical imperative to do so, given certain criteria are fulfilled. ${ }^{27}$ Was this assessment fair? I think so. Firstly, as the WHO pointed out, the EVD outbreak comprised an exceptional set of circumstances. ${ }^{28}$ The high fatality rate and rapid spread of the disease mean the risk imposed by utilising unregistered medical interventions (that do have evidence in their favour) is outweighed by the current devastation the outbreak is already creating. Furthermore, the method of controlling the disease by simply controlling its spread (a very common and age old practice) could be, and is, criticised as being ineffective in this case, with Sarah Edwards writing “WHO's previous practice of relying solely on containing the spread of EVD by restricting travel between affected villages (and now between affected

\footnotetext{
${ }^{26} \mathrm{WHO}$, Ethical considerations for use of unregistered interventions for Ebola virus disease, pg. 3.

${ }^{27}$ These criteria are outline on pgs. 5-7 of the report.

${ }^{28}$ Ibid, pg. 4.
} 
Joshua Mclvor, The Ethics of Infectious Disease Control.

countries) and by communicating health messages to promote everyday personal hygiene in the community. This 'tested' practice of the past is clearly failing this time." ${ }^{29}$ I don't think the tested practices were "failing" however. Rather, as the disease continues to spread and infect new individuals, alternative means of control must be considered, in addition to the tried and tested practices. In other words, the current approach is struggling, thus we need to be open to more drastic options, especially given the current circumstances. That said, this is not to say that continuing to impose travel restriction, perhaps clamping down harder on travel and so on, is not something worth doing. More, I think Edwards is meaning that this approach has not been sufficient and we must look at other options; in drastic circumstances we must use everything at our disposal.

Secondly, there was little commercial incentive to develop effective treatments before the 2014 outbreak, as I stated earlier. With public pressure mounting and an outbreak occurring on an unprecedented scale, health professionals were provided an opportunity to gather information on the efficacy of potential EVD treatments. Though it certainly seems distasteful to look for a silver lining when individuals are dying in their thousands from a deadly disease, the only way to study treatments for diseases like EVD is during an outbreak. This is the only way enough evidence can be gathered on the efficacy of potential treatments. Of course, tests can be done that don't involve humans but ultimately the safety and efficacy of a drug cannot be scientifically established until results in humans are studied. This further aids the case that the use of experimental treatments during the outbreak is ethically justified. Not only do individuals with no effective treatment options get the chance to utilise a treatment that may save their life but, if a comprehensive research framework is in place, many more individuals in the future may be spared the devastating consequences of the untreated disease. This point should not, and has not, gone unchallenged though. As Kevin Donovan points out "There have been examples in the past of untested and under tested therapies being rushed into service, and ultimately doing the patients a disservice. Some of these misadventures occurred on the African continent, leading to a pervasive distrust of Western drug companies using Africans as their experimental "guinea pigs"." ${ }^{30}$ Though he and many others ultimately agree with the WHO's

\footnotetext{
${ }^{29}$ Edwards, Experimental Treatments for Ebola, pg. 126.

${ }^{30}$ Donovan, Ebola, epidemics, and ethics - what we have learned, pg. 3.
} 
Joshua Mclvor, The Ethics of Infectious Disease Control.

findings ${ }^{31}$ (to varying degrees), some warn strongly against using untested drugs to combat EVD. Peter Omonzejele is one such critic and highlights some of the potential costs of using experimental drugs. He points to the 1996 Pfizer Kano meningitis trial in Nigeria. ${ }^{32}$ The trial $^{2}$ tested a new drug, Trovafloxacin, to treat meningitis. As a result of the trial 11 children died and over 200 became permanently disabled in some way. Though deaths alone may not be enough to classify a trial as immoral (cancer patients may agree to the risk the trial poses for the chance of getting better), the biggest issue was the issue of consent, or the lack thereof. ${ }^{33}$ A clinical trial is unlikely to be ethically justified if the participants are unaware of the associated risks and do not (or can't) give consent, unless the results of the trial prevented an epidemic that would kill thousands. However, considering counterfactuals like "what if the drug saved thousands but was tested unethically", become dicey when navigating the ethics of infectious disease control as they can always be raised. Ultimately, the ethical guidelines to clinical trials, such as clear consent, are designed on the basis that the desired outcome (an effective treatment) can still be achieved whilst upholding individual rights. If there were a serious possibility of saving thousands of lives that required the disregard of some of the ethical standards of a clinical trial, such as informed consent, then perhaps such action could be considered morally justified. These probabilities need to be examined on a case by case basis.

Clearly the Pfizer Kano trial had a disastrous outcome, the bypassing of ethical standards had no ultimate benefit and Omonzejele argues that the circumstances that surrounded the Pfizer Kano trial, are very similar to the circumstances of the 2014 EVD outbreak, thus "if the Pfizer Kano trial was unethical, then making experimental drugs available to West African populations who are in the midst of the EVD epidemic cannot possibly pass the research ethics test." ${ }^{34}$ This is because, as clearly demonstrated in the Pfizer Kano meningitis trial, circumventing research ethics in the quest for new drugs carries enormous risks." Omonzejele's point is well taken and he offers a very appropriate caution in a situation that, because of its unique and destructive circumstances, people are pressed

\footnotetext{
${ }^{31}$ Such as Edwards, Experimental Treatments for Ebola; Gostin, West Africa's Ebola Epidemic is Out of Control, but Never Had to Happen; and Goodman, Studying 'Secret Serums' - Toward Safe, Effective Ebola Treatments. Although each of these writers don't explicitly say they agree with the findings of the WHO panel, they all conclude the use of experimental drugs under the criteria the WHO outlined, was ethical.

${ }^{32}$ Omonzejele, Ethical Challenges Posed by the Ebola Virus Epidemic in West Africa.

${ }^{33}$ Ibid, pg. 419.

${ }^{34} \mathrm{lbid}, \mathrm{pg} .420$.
} 
Joshua Mclvor, The Ethics of Infectious Disease Control.

to do anything to help. That said I consider it extremely pessimistic to suggest that we cannot learn from a well documented and widely criticised case. Though it must certainly be ensured that there is not a repeat of the Pfizer Kano trial, we cannot allow the incident to make us overly worried that we will repeat the incident, thus prevent us from being able to provide help where possible. Additionally, let's compare the Pfizer Kano trial and the 2014 EVD outbreak to see if there are significant differences. First, it must be noted that the incident is highly controversial, with Pfizer Kano denying many of the claims made, stating: "Pfizer did not misrepresent or conceal any facts in its decision to come to Nigeria. In fact, the company's intent was clear from the beginning. Pfizer's long-term goal was to bring a lifesaving, innovative, and cost-effective form of antibiotic that could be used effectively in a meningitis epidemic in a developing country. ${ }^{\prime 35}$ Despite this, a lengthy legal battle ensued and Pfizer ultimately paid out at least some of the families. ${ }^{36}$ So bear in mind the case is not cut and dried.

The first major difference between the Pfizer Kano trial and the EVD outbreak is that there was already some effective treatment in place at the time of the Nigerian outbreak. In fact, ceftriaxone, the drug Pfizer used as the control in its study, is still the primary antibiotic used during outbreaks in Africa. ${ }^{37}$ This is clearly a big difference. Furthermore, it is even alleged that the NGO Médecins Sans Frontières was offering the standard treatment in another part of the same building as the Pfizer Kano trial. ${ }^{38}$ The central point here being: there was already a standard effective treatment in place for meningococcal meningitis at the time of the Pfizer Kano trial. As we well know, this was not the case with the 2014 EVD outbreak. Had there been no effective treatment at the time of the Pfizer Kano trial, and their drug had at least saved some lives, would we still have deemed it unethical? My intuition is; we would have looked at it through a much brighter lens, looking at the lives that were saved, not the ones that were lost. A second big difference between the Pfizer Kano trial and the EVD outbreak, is the fatality rates of the diseases. As said on page six of this thesis, EVD has a case fatality rate averaging on 50\%, whereas meningococcal

\footnotetext{
${ }^{35}$ Pfizer, Trovan, Kano State Civil Case - Statement of Defence, pg. 1.

${ }^{36}$ The Guardian, Pfizer pay out to Nigerian families of meningitis drug trial victims.

${ }^{37}$ WHO factsheet, Meningococcal Meningitis, pg. 2.

${ }^{38}$ Wikipedia, Abdullahi v. Pfizer, Inc.
} 
Joshua Mclvor, The Ethics of Infectious Disease Control.

meningitis has a fatality rate of $5-10 \%$ (with treatment). ${ }^{39}$ EVD simply kills more infected individuals that meningococcal meningitis, which means the survival rate of any given infected person, is much worse. Thus, the use of experimental treatment may be seen as more rational from an individual's point of view: they are more likely to die if untreated anyway, so why not take a chance with the experimental treatment? On the other hand the meningococcal patient might rather take their chances with the standard treatment and not risk the unknown effects of the experimental treatment.

The final difference between the Pfizer Kano trial and the EVD outbreak is that meningococcal meningitis has no animal reservoir and is a stage five disease. ${ }^{40}$ This means there is much more opportunity to study the disease in humans and develop treatments more frequently. Remember one advantage of studying experimental treatments for EVD in humans during an outbreak, is that the disease often disappears for a long time in between outbreaks, as it retreats back to its animal reservoir. There was no such imperative during the Pfizer Kano trial. So, to rebut Omonzejele's concern that the use of experimental treatment during the EVD would result in a repeat of the Pfizer Kano (which was at least controversially unethical), I think the above differences mean we need not worry about falling into unethical territory. Furthermore, the conditions outlined in the latter part of the WHO report, if met, would ensure the highest ethical standards are upheld. It is always difficult to strike a balance between providing help as quickly and efficiently as possible, and ensuring the principle of first doing no harm is upheld. In this case, I think the WHO have gotten it right. The nuance is well summarised by Jesse Goodman: "As we move forward, quickly but cautiously, in using and testing new therapies, we have already learned some lessons from this outbreak - regarding the need to build trust, the need to enhance public understanding of experimental treatments and their safe evaluation, and the critical nature of the capacity both for public health intervention and to ethically field clinical studies under challenging conditions. When it comes to infectious diseases, we are increasingly one world and dependent on each other for knowledge, safety, and security." ${ }^{\prime 1}$ Extrapolating this to our case here, this means we have collectively learned some important ethical lessons

\footnotetext{
${ }^{39}$ WHO factsheet, Meningococcal Meningitis, pg. 2.

${ }^{40}$ Referring again to: Wolfe, Dunavan, \& Diamond, Origins of major human infectious diseases.

${ }^{41}$ Goodman, Studying 'Secret Serums' - Toward Safe, Effective Ebola Treatments, pg. 1088.
} 
Joshua Mclvor, The Ethics of Infectious Disease Control.

through experience and we can be optimistic that the use of experimental treatment in the EVD outbreak will be done in a safe and ethical manner.

The panel did not arrive at a decisive conclusion as to the ethics of using experimental treatment as prophylaxis in any case. With the limited experimental treatments available at the time, I imagine the need for prophylactic treatment would be limited and its use not warranted. There was some discussion, though opinions were split on the issue, of prioritising health care workers as candidates for the use of therapy. At any rate, it would not appear as though any clear outcome was made with regards to the use of experimental treatment for strictly prophylactic purposes and I am unsure if this ultimately occurred. For now, we must consider questions $1 \mathrm{~b}$ and $1 \mathrm{c}$, listed above, as unanswered by the WHO. ${ }^{42}$ For our purposes, I would consider the use of an experimental drug as prophylaxis to come down to a few factors. First, is how much of the drug is available. If there is very limited availability of the drug, then the priority should be to aid those who are sick. This has the benefit of both helping those who otherwise may die, and providing clinical information on how the drug works as a treatment. Second, assuming availability wasn't an issue, I would consider who should receive the drug as prophylaxis. Intuitively, front-line healthcare workers would benefit the most from such protection and, as health care workers; they would understand the risks associated with using an experimental drug, thus minimising issues of consent. Finally, we must consider what other means of protection from infection there are. If cheaper alternatives protect individuals from the disease equally or almost as well as the drug would, then it would be an unnecessary waste of resources to widely use the drug as prophylaxis. Assuming the above factors allow for the use of an experimental drug, and all the WHO's criteria are met, prophylactic use of an experimental drug is ethical. Unfortunately, in the case of the 2014 EVD outbreak, there wasn't enough availability of the drugs to ethically allow for prophylactic use. ${ }^{43}$

I do not wish to spend much time discussing the WHO's answer to question 2, listed above. In relation to the EVD outbreak, I agree with the WHO's conclusions as to the

\footnotetext{
${ }^{42} \mathrm{WHO}$, Ethical considerations for use of unregistered interventions for Ebola virus disease.

${ }^{43}$ Furthermore it does not appear as though Zmapp, the drug mentioned earlier that was used to treat the American doctors, had prophylactic capabilities. A brief commentary on the drug is: Tracey ZMapp and Ebola [link in references]. Therefore, the question of using experimental treatments as prophylaxis was purely theoretical at the time of the outbreak, though we can certainly consider it.
} 
Joshua Mclvor, The Ethics of Infectious Disease Control.

conditions that must be met in order for the use of experimental treatments to be considered. Any deviation in my own opinion from the WHO's conclusions applied beyond EVD, will be discussed in section two of this paper. For now, the reader can refer to the WHO report to find the full, detailed list of conditions, which include: sticking to wellfounded traditional research ethics, utilising risk assessment, utilising experimental treatments that have been proven safe in relevant animal models, transparency, clear consent, the sharing of data and information between agencies, and feasibility. Thus, as I stated earlier, we can be optimistic that in this particular case, the correct ethical measures should be taken and we won't have a repeat of the Pfizer Kano trial.

Finally, with the experimental treatments being a particularly scarce resource, how should we prioritise the allocation of the treatment? On this particular point the WHO recommended further discussion was warranted. They also suggested a further discussion was allocated to answer the question: what are the ethical criteria for distributing the experimental treatments among communities and between countries? ${ }^{44}$ The WHO's own response to the point was understandably limited; the issue is contentious and requires a lot of philosophical debate. Furthermore, there was disagreement on what I consider to be an important point and I suspect the reason the report's analysis on the topic is more or less inconclusive, is due to a lack of unanimity after the panel discussion. I will now take a look at the WHO's comments and offer some analysis of my own. The first criterion for ethical distribution that the panel mentions is "Distributive justice: fairness between countries and among populations within countries". ${ }^{45}$ On the surface, this might seem an intuitively valid criterion, but I personally think a lot hinges on what our concept of "fairness" is, as well as what the scope of the "countries" is. Though fairness may seem like a simple concept, what is fair is often interpreted in many different ways. For example, we may consider that if there is a joint expenditure to be made between a group of five friends, then the fair thing to do would be to make them all pay an equal amount. Yet what if two of those friends are low income students, accruing debt by the week, whilst two work full time, minimum wage jobs, and the final friend recently won the lottery? If all pay an equal amount, the proportion the amount will be relative to total worth or income will differ greatly between

\footnotetext{
${ }^{44}$ WHO, Ethical considerations for use of unregistered interventions for Ebola virus disease, pg. 6.

${ }^{45}$ Ibid.
} 
Joshua Mclvor, The Ethics of Infectious Disease Control.

friends, thus affecting some of the friends more negatively than others. Perhaps, it may be argued, it is still fair because the friends who can't comfortably afford the expenditure shouldn't partake in the joint group activity that is incurring the cost in the first place. But where does that end? It is not as if the friend that won the lottery had to work for his money, he was just lucky. The answer to this thought experiment doesn't matter, what does matter is that people probably differ greatly on what they consider to be the right answer to what the 'fair' approach is in the thought experiment. So we must be clear about what is considered fair, when it comes to distributing the experimental treatments across countries. I would argue the fairest approach would be to send the most treatments to those countries that stand the highest chance of benefiting the most people and send the least treatments to those countries where the use of the treatments may be in vain (i.e. largely ineffective at preventing the spread of the disease or benefiting large portions of the population). So in the case of the 2014 EVD outbreak, the limited drugs should have been sent to the West African countries that were affected.

In the case of EVD the issue of how to distribute the limited number of experimental treatment doses between countries/societies may be relatively clear cut. Only 36 cases of EVD were reported outside of the main three West African nations, and the threat to other countries was relatively low, due to such advanced warning. This would not necessarily be the case for other possible pandemics, so the issue would have to be answered on a caseby-case basis. Factors such as how many doses are available, how many individuals are affected, and the biological features of the disease, are going to play a big role in determining the most ethical way to distribute the experimental treatment between countries. When the number of available doses becomes few enough, the answer to the issue becomes relatively arbitrary, especially if many people across a few countries are affected: there simply aren't enough doses to significantly hinder the spread of the disease so whether or not a particular country receives doses almost becomes a lottery. In such a case you can either split the doses between countries proportionally, based upon number cases, or begin looking at the individuals within each society. This method can unfortunately appear ruthlessly pragmatic, yet it is what is necessary and fair when it comes to controlling infectious diseases. 
Joshua Mclvor, The Ethics of Infectious Disease Control.

How to distribute experimental treatment within a society comes down to two criteria, outlined by the WHO panel: "Reciprocity and Social Usefulness". ${ }^{46}$ Essentially, this meant some members of the panel proposed health care workers to be of the highest priority when it comes to receiving experimental treatment, because they have risked their lives treating the ill (thus priority would adhere to the principle of reciprocity) and they are very important when it comes to controlling the outbreak and helping others (this would adhere to the principle of social usefulness). The report notes that "Other panel members advocated that the patients in the community should have the same priority as the groups mentioned above [health care workers], particularly for therapy." ${ }^{47}$ This is fundamentally an example of a disagreement over what is considered 'fair', as discussed above. Furthermore, this disagreement in the panel raises the issue of why Western healthcare workers were being treated with experimental drugs, whilst Africans were being left to die. It's a contentious point, but I agree with the panel members that supported the prioritisation of health care workers.

The backbone to dealing with outbreaks in any corner of the globe is always going to be health care workers on the ground. As a society, we need to be able to assure these people that their altruistic attitude in helping others at great personal risk will be reciprocated if they fall ill. To do otherwise runs the risk of discouraging those that may be of great help, from offering to aid in outbreaks. That said, this should include African health care workers who have suffered greatly throughout the outbreak while continuing to risk their lives to help others. ${ }^{48}$ As the health care infrastructure in the affected countries was far from adequate to deal with such an outbreak, it may be of more importance to aid those systems and workers of the affected countries. That way they are better able to cope on their own, which may be a better result than initially offering experimental treatment to Western health care workers, unless if by doing so more resources are freed up to aid the local community and health care system. ${ }^{49}$ Furthermore, health care workers are better educated on the risks associated with undergoing experimental treatment and so are better

\footnotetext{
${ }^{46} \mathrm{WHO}$, Ethical considerations for use of unregistered interventions for Ebola virus disease, pg. 6.

${ }^{47}$ Ibid.

${ }^{48}$ The WHO has published a report on the incidences and casualties of health workers in the afflicted countries that highlights the sacrifices the health community in the afflicted countries have made: WHO, Health worker Ebola infections in Guinea, Liberia and Sierra Leone.

${ }^{49}$ Kevin Donovan tackles this and other related questions excellently in his paper: Donovan, Ebola, epidemics, and ethics - what we have learned.
} 
Joshua Mclvor, The Ethics of Infectious Disease Control.

candidates to provide full consent. Ultimately, though we may be tempted to consider regular members of the community as equal candidates for experimental treatment, I think there are strong pragmatic reasons why we need to consider health care workers as higher priorities for experimental treatment. A strong health care system, which will be founded on healthy and able workers, is necessary to effectively combat any infectious disease, thus prioritising health care workers for experimental treatment is necessary in combating EVD.

This conclusion, however, does not get us across the finish line. We still must tackle the issue of how to prioritise between western healthcare workers and local African healthcare workers. If two American healthcare workers received ZMapp after contracting EVD, why shouldn't local healthcare workers receive the same? Fundamentally, should the visiting Swedish healthcare worker get prioritisation over local healthcare workers? It's a hard question to answer, but I think the criteria of reciprocity and social usefulness, discussed above, may help us here. First, let's examine a pragmatic concern: rational selfinterest. The Swedish healthcare worker volunteering in Guinea is choosing to put her own health at risk to battle a disease that is not affecting her home country and is unlikely to anytime in the near future. Could we be disincentivising such altruistic action, and thus a means to combat the disease, by openly giving local healthcare workers what few doses of potentially life-saving experimental treatment there are over foreign healthcare workers? It's a valid concern yet a hard one to answer. On the one hand, it may very well be the case that we could be disincentivising such altruistic action in the future if we do not prioritise foreign healthcare workers when it comes to doses of experimental treatment. Yet, I would be surprised if this were the case. The healthcare workers in question are already knowingly risking their lives, many before they even knew of experimental treatment like ZMapp, so I don't think such prioritisation would necessarily dissuade them. Furthermore, the same argument could be made for local healthcare workers. If foreign healthcare workers were prioritised, then local doctors and nurses might choose to stay at home and avoid potentially contracting the disease rather than risk their live. But once again, I am dubious that this would be the case purely as a result of prioritisation of foreign doctors for doses of, say, ZMapp. So I cannot see this being a persuasive factor in either direction. Therefore we must look to reciprocity and social usefulness. Are either party being more fairly compensated by receiving experimental treatment prioritisation? I would argue it is 
Joshua Mclvor, The Ethics of Infectious Disease Control.

marginal. Though the foreign healthcare workers are probably going more out of their way to help where they otherwise would be one hundred per cent safe, the disease could not be fought without the massive effort made by local healthcare workers. What of social usefulness? This would be hard to quantify, though perhaps healthcare services may have a chance at it. On average do foreign healthcare workers or local healthcare workers do more when it comes to battling EVD? How do we even quantify this: patients treated, number of house spent in "at risk" environments, "expertise" brought to the field? I would be surprised if proportionally, this was not pretty close to even. If there were a strong difference, then I would suggest prioritising treatment accordingly. Otherwise, the issue isn't how to prioritise between foreign healthcare workers and local healthcare workers, but how to prioritise within the category of healthcare worker across the board. The doses should be spread between foreign and local healthcare workers, but prioritisation should go to those who stand to be able to do the most social good if they were to survive. Therefore, the local doctor with twenty years expertise working with in the local environment with deadly diseases, who has mastered infectious disease control techniques and who's methods have saved countless lives, should probably get priority over the foreign healthcare worker who is acting as a stretcher-bearer or decontaminator. If the doctor lives, he is likely to generate more utility than the stretcher-bearer. This may seem ruthlessly pragmatic, but it is rational. This logic would have to be applied across the entire healthcare worker body, in all three countries, especially when the doses are so low.

The remaining criteria for prioritisation of experimental treatment that the WHO panel's report provides are seemingly uncontroversial. They include; transparency in the allocation process, a duty to provide supportive care, a minimum amount of infrastructure must be in place, involving the community, consent, and promoting trust in the health care system..$^{50}$ I can't imagine a substantive argument could be made against these criteria, as most are pragmatic.

This concludes my discussion of the ethics of utilising experimental treatment during the EVD outbreak. The main purpose of the discussion was to highlight the complexity and controversy that surrounds even apparently simple ethical decisions, during a difficult situation like an outbreak. Additionally, the question of the use of experimental treatment

\footnotetext{
${ }^{50} \mathrm{WHO}$, Ethical considerations for use of unregistered interventions for Ebola virus disease, pg. 6.
} 
Joshua Mclvor, The Ethics of Infectious Disease Control.

will arise again and I want the reader to have a comprehensive understanding of the logic and argumentation involved in justifying any use of experimental treatments. As I stated earlier, many people may have considered the answer to the question obvious; of course it is ethical to offer experimental treatment during an outbreak. But the issue is a lot more complex than that and in reality we really only scratched the surface here: delving in to just a few key ethical concerns. What I encourage the reader to keep in mind, is the overall plan for this thesis. We are going to move from the very specific case study of the 2014 EVD outbreak, to more general ethical principles of infectious disease control. So, we need to keep in mind how the lessons we are beginning to learn from the EVD outbreak may apply to other scenarios and perhaps more importantly, how they may not apply to different scenarios.

\subsection{An ethical issue: quarantine.}

The final ethical issue I would like to consider before moving on is the issue of quarantine and isolation. This will be a brief look, as the ethics of quarantine and isolation will be discussed in greater detail later on in this paper, as it is one of the oldest and most common means of dealing with infectious diseases. Furthermore, it involves the physical restriction of one's autonomy, which is no small freedom to take away. During the EVD outbreak there were some worrisome quarantine practices, to say the least. As such, it is worthwhile taking a quick look at some of the issues that arose from quarantine during the outbreak.

As previously stated, when EVD struck in 2014, there was no effective treatment in place and the countries it spread through had poor health care infrastructures. This meant one of the only tools available to try and control the spread of EVD, in many cases, was quarantine. Quarantine is the practice of physically isolating individuals who are suspected to have an infectious disease or who have come in contact with someone who has an infectious disease, in order to prevent the spread of the disease. This is different from isolation, in which individuals who are already confirmed with having a particular infectious disease are physically isolated. It is worth noting that already we can see one major problem when it comes to applying quarantine to the EVD outbreak: individuals are not contagious until they are symptomatic. So although asymptomatic individuals may carry the disease, they will not be able to spread it to others until they are symptomatic. So in theory, most of 
Joshua Mclvor, The Ethics of Infectious Disease Control.

the focus in the case of EVD should be on isolating individuals once they are symptomatic and thus confirmed to have the disease. Though quarantining those who may have been exposed to the disease will certainly provide a further safety net and allow for immediate isolation of symptomatic patients, the monitoring and isolation of sick individuals may prove just as effective, yet less invasive and restrictive as quarantining large groups of individuals who may be infected. Though of course precautions and pragmatic concerns will inevitably call for the quarantining of individuals, the scale and tactics of some of the quarantining practices during the EVD outbreak was bordering on medieval. ${ }^{51}$ This brings us to one of the biggest concerns regarding the ethics of quarantine during the EVD outbreak: adherence to the principle of reciprocity. This principle will be talked about in more detail later but the idea is: given quarantine is supposed to be for the benefit of the society and comes at a cost to the individual, individuals who are quarantined are ethically required to be treated properly, with due respect and gratitude for being isolated. In some parts, during the EVD outbreak, no such reciprocity was given. Lawrence Gostin summarises:

"They have invoked quarantine, ranging from stay-at-home days for "reflection, education, and prayers" to guarded home confinement. The military has been deployed for house-to-house searches, traveller checkpoints, and cordon sanitaire (a guarded line preventing anyone from leaving)-sometimes separating people and regions of the country.

These cordons sanitaires are of the medieval variety practiced during the Black Death. In West African "hotspots" (with uncontrolled transmission) armed troops have established blockades, closed roads, and banned travel beyond the guarded perimeter. The populace is fearful, not only of exposure to EVD, but also of isolation and starvation, as food prices soar. ${ }^{152}$

Not only do countless ethical concerns come to mind when examining these practices, but there are major practical concerns as well. If individuals are treated in the manner outlined above, then people will become fearful of government interventions. They will be dubious when genuine help does arrive, for fear of being treated like plague sufferers in Victorian England. They will be reluctant to go to hospitals, for the treatment of EVD or indeed any other illness, out of fear of being forcibly placed in areas where they run the risk of catching EVD, if they haven't already. This is hugely problematic if we want an effective response to be taken, as any response will require the trust of the public. If the government

\footnotetext{
${ }^{51}$ Gostin et al., West Africa's Ebola Epidemic is Out of Control, but Never Had to Happen, pgs. 3-4.

52 Ibid.
} 
Joshua Mclvor, The Ethics of Infectious Disease Control.

doesn't have the public's trust, for whatever reason, then it will be difficult to get them to comply with practices which will objectively benefit them. So, practical concerns aside, how do the practices, described above, hold up to ethical considerations? Could they ever be ethically justified?

To answer the above questions, I think we need to imagine potential pandemics as falling onto a scale, based upon biological factors of the disease in question and social, political, and economic factors that surround the outbreak. On one end of the scale we have increased incidences of the common cold, not too many but enough to classify as an outbreak or pandemic. Would cordoning off entire communities, cutting them off to food, water, and medical care, be warranted if it was effective in preventing the spread of the disease? Probably not, in all likelihood a lot more harm than good would come of it. On the other end of the scale, we have a disease that kills upwards of $50 \%$ of those it infects, and a further $45 \%$ become delusional, angry, lose touch with all sense of self, and have an overwhelming desire to consume human flesh. Would it be ethically justified in this case to, say, employ the military to blow up the bridges to an entire city, cutting it off completely to the outside world with a policy of shooting on site anyone who tries to escape? It may very well be ethically justified, and I would argue it would be if it prevented the spread of a disease which was threatening the entire planet. Furthermore, in such extreme circumstances we as a society can still strive to uphold the principle of reciprocity to the best of our ability. Food drops and exit points through which individuals can get tested and let through if they are shown to be clear of the disease, could be ways in which to make the horrendous situation just that much better for those affected. It is an extreme example, but worth considering.

Whilst ethical disease control involves upholding a whole host of ethical principles, sometimes the reality of the situation calls for more drastic measures, in order to maximise utility and save more lives. Pragmatic concerns are very much a part of ethics and are the foundation of the use of quarantine in the first place. So how does the quarantining during the EVD outbreak hold up to ethical scrutiny? Well, it is hard to make a blanket statement, but insofar as the types of quarantining described by Gostin above: not too well. Though the quarantining of communities may very well have prevented the further spread of the disease and thus be justified, more could have been done to uphold the principle of 
Joshua Mclvor, The Ethics of Infectious Disease Control.

reciprocity. Food and medical supplies could have been transported in a controlled way through the cordons, to aid those inside. Individuals who have been reported as very ill could have been retrieved by properly protected medical staff, and brought to isolation in hospitals. If those inside the cordons were being quarantined to prevent the disease spreading and ravaging even more of the population, then much more effort should have been put towards ensuring these people were looked after properly and that the harm to them was minimised. That said, as we well know the healthcare infrastructures of the affected countries was poor and they may well have been doing all they could with what resources they had. The conclusion is: from an outside perspective it appears as though the quarantine methods they employed could have been better and they could have done more to uphold the principle of reciprocity.

This subsection was a very brief look at the ethics of quarantine during the EVD outbreak. It is important that we can learn from situations like that discussed above, as until we develop better strategies, quarantine and isolation will be an important aspect of infectious disease control, especially during outbreaks. We can keep this case study in mind when looking at other cases of quarantine later.

\subsection{Conclusions: Lessons from the EVD outbreak and moving forward.}

In this section we have looked at the West African EVD outbreak of 2014. We used the EVD outbreak as a case study to examine some of the ethical questions that can be raised in such scenarios, as well as critically assess some of the measures which were taken to control the outbreak. We took an in depth look at the ethics of the use of experimental treatment during the outbreak and highlighted some of the difficulties in addressing such an issue, which may not always be immediately apparent. Additionally, we had a quick look at the ethics of quarantine, notably how the carrying out of quarantine was very likely unethically conducted during the EVD outbreak. It is my hope that by looking at the EVD outbreak in some detail, and analysing some of the ethical questions it raises, we can now start to think about how the ethical conclusions we arrived at can be applied to other infectious diseases outbreaks. Armed with a real world sense of some of the difficulties that surround the ethics of infectious disease control, we can now begin to apply our thinking in a broader sense and to other scenarios. But, as we move forward into more theoretical territory: what of the EVD situation? How is the global health community moving forward? 
Joshua Mclvor, The Ethics of Infectious Disease Control.

As of this writing, reported cases of EVD have dropped to zero. Human to human transmission of the disease has said to have ended in all three affected countries. ${ }^{53}$ The worst is over with respect to this outbreak and we can now look to how we can better manage such an outbreak in the future. For a start, with the outcome of the August $11^{\text {th }}$ 2014 WHO panel concluding that it was ethical to use experimental treatments during the EVD outbreak, there is now a very promising vaccine candidate. The WHO states: "Results from an interim analysis of the Guinea Phase III efficacy vaccine trial show that VSV-EBOV (Merck, Sharp \& Dohme) is highly effective against Ebola." ${ }^{54}$ This is very promising and it would seem as though viewing the outbreak as an opportunity to study the disease and develop treatment for it, has hopefully paid off.

The WHO published an article in January 2015 that outlined what needs to happen in 2015 and what we can learn from $2014 .^{55}$ Though slightly out-dated now, the article still offers many relevant commentaries as to what the global health community and the local health communities of those affected countries can learn from the outbreak. First, as we have already discussed, the global community has suffered a harsh lesson as to what can happen when poor countries with weak health systems receive a blow such as an outbreak of EVD: the healthcare systems collapse. This fact has sparked some, like Lawrence Gostin, to suggest the creation of a "WHO "Health System Fund"”. ${ }^{56}$ This fund would be used to rebuild and strengthen the healthcare infrastructures of those affected countries, as well as their at risk neighbours. It is clear that when considering potential future outbreaks, it may be of global interest to invest in developing poorer countries' health care infrastructures, as this will help prevent future outbreaks from spreading across countries; from becoming a global pandemic.

Second, preparedness for a disease can make a substantial difference in defending against an outbreak. The WHO article lists the cases of Nigeria, Senegal, and Mali, who unlike the affected West African countries, were ready for the EVD outbreak (in no small part because their neighbours were already in the grips of an outbreak). ${ }^{57}$ This preparedness allowed the countries to prevent EVD from gaining a foothold in the population. The need

\footnotetext{
${ }^{53}$ Current reports on EVD in West Africa can be found on the WHO website at: http://www.who.int/csr/disease/ebola/en/

${ }^{54} \mathrm{WHO}$, World on the verge of an effective Ebola vaccine.

${ }^{55}$ WHO, Ebola response: What needs to happen in 2015.

${ }^{56}$ Gostin, West Africa's Ebola Epidemic is Out of Control, but Never Had to Happen, pg. 6.

${ }^{57}$ WHO, Ebola response: What needs to happen in 2015.
} 
Joshua Mclvor, The Ethics of Infectious Disease Control.

for preparedness may support calls from those such as Diamond et al. to create an "early warning system", so that we are not blindsided by potential outbreaks in the future; or can at least make cost/benefit analysis judgements based upon the threat of various diseases. ${ }^{58}$

Third, we must take a multi-faceted approach when trying to control infectious diseases. No one measure or tactic will do it all, thus we must be ready to tackle the disease on multiple fronts. Furthermore, though this is not expressly mentioned by the WHO here, when we are taking a multi-faceted approach to infectious disease control, we must be aware that we are more likely we are to stumble into ethically treacherous areas, as the number of factors that need control and consideration increase. It is exactly when we intend on tackling the disease to the best of our abilities, that we must also be weary of neglecting our ethical analysis.

Finally, the WHO suggests that "community engagement is the one factor that underlies the success of all other control measures. It is the linchpin for successful control. Contact tracing, early reporting of symptoms, adherence to recommended protective measures, and safe burials are critically dependent on a cooperative community." ${ }^{59}$ This boils down to the fundamental principles of transparency and reciprocity that are not only of ethical importance, but also pragmatic importance. A community will not comply with disease control measures if they are not actively informed and involved in the rationale behind the measures, and treated with due respect.

These are just a few of the major lessons that we have learnt from the EVD outbreak in West Africa. Moving forward, it will be important to keep these in mind, as well as their real world context, as we move on to a more theoretical approach to the ethics of infectious disease control.

\footnotetext{
${ }^{58}$ Wolfe et al., Origins of major human infectious diseases, pg. 283.

${ }^{59}$ WHO, Ebola response: What needs to happen in 2015.
} 


\section{Section Two: Do the ethics of infectious disease control vary depending on the disease?}

\subsection{Introduction.}

After our discussion on the 2014 EVD outbreak in West Africa, it may be tempting to believe that we have learnt something ubiquitous when it comes to the ethics of infectious disease control, or something substantially profound. But I think we must be careful here, because this is not entirely the case. Though we have certainly learnt something substantial from the EVD outbreak about the ethics of infectious disease control and we may be able to apply some of these lessons to other cases (as we should), we mustn't get complacent in believing we necessarily have a greater understanding of how to deal with any future infectious disease epidemic. Will the ethical conclusions we have arrived at in regards to the use of experimental treatment during an outbreak apply to all other infectious diseases? What about in regards to rhinovirus, the common cold? Would it be ethical to use experimental treatment if there was an outbreak of the common cold in a population? I think most of us would have the intuition that it is not okay to use experimental treatment during a rhinovirus outbreak. Why is this? Because of how the disease is spread, it's commonality, or perhaps its lethality? Now this is an extreme example, the common cold is clearly a world apart from EVD; they are not really in the same league. Nonetheless, there are countless infectious diseases out there and we can know they present a range of modes of transmissions, severity, and so on. So, if we can imagine that there is a substantial difference in how we approach some of the ethical conundrums with regards to EVD versus rhinovirus, then we can imagine that there may be more subtle, yet nonetheless important, differences in how we approach some of the ethical conundrums with regards to diseases that are more similar to EVD, yet not identical.

It is these differences I would like to examine in this section. I will be looking at some of the arguments generally put forward when considering the ethics of infectious disease control. I want to examine some general principles often discussed, such as the principle of reciprocity, and see how these fare when considering various factors of different infectious diseases. From here, I hope that an image may begin to form, of certain epidemiological and microbiological factors of different infectious diseases, which will play a major role when it 
Joshua Mclvor, The Ethics of Infectious Disease Control.

comes to considering the ethics of infectious disease control. Ultimately, the goal of this section is to perform some of the philosophical leg work required to support a flexible and adaptive ethical framework that could be applied to health care policy. More generally, I hope to show that we cannot take a 'one size fits all' approach when it comes to considering the ethics of infectious disease control. We must recognise that the differences in physical factors of infectious diseases will affect our approach to controlling the diseases in an ethical manner; more than may be first apparent.

\section{$2.2 \mathrm{~A}$ starting point: the Andrew Speaker incident.}

I would like to start our discussion with a case study, which will be referenced throughout the rest of the paper. The case study asks what the government's powers should be when it comes to restricting individual liberties in order to control the spread of infectious diseases. The case study is known as the "Andrew Speaker Incident". I will be using Fidler et al.'s useful summary of the incident and the reader may wish to read the full article for a more in depth summary of the incident. ${ }^{60}$ All credit goes to the writers of that paper for the information provided in this case study.

In January 2007, a U.S. lawyer named Andrew Speaker went to the doctor to undergo a chest x-ray and CT scan, which revealed that he had an abnormality in his lungs. However, other concurrent tests were inconclusive so he underwent follow up medical testing in March. A diagnostic bronchoscopy revealed Speaker had tuberculosis (TB) and he was prescribed the standard first-line drugs usually used to treat TB. While Speaker's tests (biological samples) were sent for further analysis in a laboratory, Speaker advised the Fulton County TB clinic that he had plans to go overseas in May and as such the clinic requested that the laboratory analysis being done on Speaker's TB isolate be hurried. Samples were also sent to the Centers for Disease Control (CDC) to have additional testing carried out. Before leaving in May; Speaker, his private physician, and his family met with the Fulton County Health Department (FCHD) to discuss his TB infection. Due to the hurried laboratory testing, it had been revealed the day before that Speaker actually had multidrug-

\footnotetext{
${ }^{60}$ Fidler et al., Through the Quarantine Looking Glass: Drug-Resistant Tuberculosis and Public Health Governance, Law, and Ethics, pg. 617.
} 
Joshua Mclvor, The Ethics of Infectious Disease Control.

resistant tuberculosis (MDR-TB), which, as the name suggests, means that Speaker's strain of TB was resistant to multiple of the first line drugs used to treat TB. Therefore, during Speaker's meeting with the FCHD, he was told not to go travelling overseas. In the subsequent days the FCHD, Georgia Department of Public Health (GDPH), and the CDC began to discuss legal options for restricting the travel of someone with MDR-TB.

When the FCHD tried to locate Speaker, they could not find him. Unknown to anyone, Speaker had accelerated his travel plans and had already left on his overseas travels to Europe. As the CDC began attempts to locate Speaker in Europe, further testing came back that revealed Speaker in fact had extensively drug-resistant tuberculosis (XDR-TB). XDR-TB is worse than MDR-TB in that Speaker's particular strain of TB was not only resistant to multiple frontline drugs normally used to treat TB, but also resistant to many of the second line drugs used in the case of MDR-TB. This sparked a nation-wide border alert for Speaker.

The CDC eventually managed to track Speaker down in Rome and informed him of his XDR-TB diagnosis. They advised him not to travel on any international aircraft due to the threat he posed to other people. Despite Speaker indicating to the CDC that he would stay in Rome, he ended up flying to Prague and then to Montreal. When they discovered Speaker missing yet again, the CDC requested that the Transportation Security Administration (TSA) prevent him from boarding any plane bound for the U.S. and they informed the Italian Ministry of Health as well as the WHO of the situation.

Eventually, on the $25^{\text {th }}$ of May, Speaker drove back into the U.S. from Canada and despite the border guard being aware of the border alert; Speaker was let back into the United States. Eventually locating Speaker via cell phone in New York State, the CDC ordered Speaker to drive to a hospital where he would be put under federally mandated isolation. When he arrived at the hospital, Speaker was issued with a provisional federal quarantine order, the first issued since 1963. Now that Speaker was isolated and further testing and assessment had begun, the CDC began tracking down those with whom Speaker came in close contact, including those who flew on the same planes as him. This required major international cooperation. 
Joshua Mclvor, The Ethics of Infectious Disease Control.

In the subsequent months, Speaker moved around a few hospitals for treatment and his story began to attract attention in the media. Questions were raised about how such an incident had occurred, what had gone wrong, and who was to blame. Furthermore, some of the passengers who travelled with Speaker from Prague to Montreal initiated tort litigation against Speaker. After surgery was performed to remove some of the TB from Speaker's lungs, Speaker was eventually declared non-contagious at the end of July 2007.

Controversy continued for long after Speaker was declared non-contagious. There were discrepancies in the testimonies of Speaker and the CDC. For example, Speaker indicated he was never told of the substantial risk he posed to other travellers prior to leaving the United States. ${ }^{61}$ Furthermore, later testing showed that his initial diagnosis of XDR-TB was false and he only had MDR-TB. The spotlight was on the CDC as to how such an incident could arise, posing a threat to thousands of individuals around the world. In this case, the world was lucky, but had the disease been more contagious and lethal, there could have been a real potential for a serious pandemic. The holes in public policy regarding infectious disease control were brought to the forefront and people began to think about the idea of mandatory isolation. The ethical questions of infectious disease control were brought into the public domain.

\subsection{The foundation of infectious disease control: the principle of reciprocity.}

The Speaker incident clearly highlighted the importance of having effective infectious diseases control policy in place, in order to manage and prevent potential outbreaks. That said, beyond the simple pragmatics of needing such policy, how do we ensure we are acting in an ethical manner when enacting infectious disease control policy? It is this question we will turn our attention to now. In this subsection, we will begin with some of the more fundamental questions of the ethics of infectious disease control, and move on from there. There is no right or wrong place to begin when discussing the ethics of infectious disease control, but I will try to start where I think it is most intuitive, and go from there.

I will begin our discussion with a paper that highlights some of the most basic questions in the ethics of infectious disease control and underscores the importance of

\footnotetext{
${ }^{61}$ ABC News, Exclusive: TB Patient Asks Forgiveness but Defends Travel.
} 
Joshua Mclvor, The Ethics of Infectious Disease Control.

asking the question: "Is there a moral obligation not to infect others?"62 The paper covers three major points: do we have an obligation not to spread contagions, would such an obligation apply to, say, the workplace, and does the mode of transmission of the disease affect the ethics? We are not too interested here with the specifics of moral obligations not to spread infectious diseases in the workplace. I will view that particular question as more of an example, thus combining the first two points into one and start from there. While I think most of us would accept that it was immoral of Speaker to willingly risk infecting others with TB (assuming he indeed knew the risks), many may be more reluctant to say that it is likewise immoral to risk infecting others with the common cold. Yet I think we can all accept that to contract the common cold, is to be harmed (if not much less than with some other infectious diseases). Harris and Holm put it this way: "If communicating a disease is to inflict a harm proportionate to the severity of the disease and its consequences then the same moral obligation not to inflict such harm on others applies as much to disease as it does to other knowingly inflicted harms and applies as much in the social context as it does at work." ${ }^{63}$ In order to move forward here, I think we must accept Harris and Holm's claim that communicating a disease is to inflict harm. If one was to fall ill with any infectious disease, they have clearly suffered some form of harm. If the person transmitting the disease had not done so, the recipient of the disease would not have fallen ill and thus suffered accordingly. Putting fault or moral obligations aside (for now), we can certainly say harm has been caused. Perhaps an argument could be put forth against this claim (I couldn't imagine one), but we will not try to justify this point further as the claim is not a big one and seems intuitively correct. So, to be the recipient of an infectious disease is certainly to experience something harmful and this harm will vary depending on the severity of the disease.

So, if infecting someone with a disease is indeed to cause harm, how do we understand the blameworthiness of the vector (the person transmitting the disease) of the disease? What measures must one take in order to prevent the spread of an infectious disease? If we expect individuals to never willingly run the risk of infecting others with a disease, then it would seem as though some pretty extreme and medieval measures may be justified when it comes to preventing individuals from spreading disease. Yet this seems obviously absurd. I will paraphrase what Harris and Holm have to say on the issue. If

\footnotetext{
${ }^{62}$ Harris \& Holm, Is There A Moral Obligation Not To Infect Others?

63 lbid, pg. 1215.
} 
Joshua Mclvor, The Ethics of Infectious Disease Control.

someone contracts a cold or flu and this person works in an office space that is relatively tightly spaced, as many offices are, then that person can reasonably expect to infect one or two others when they go to work (or at least run a very high risk of this occurring). ${ }^{64}$ Yet, if the person were to stay at home they can completely avoid the risk of infecting others, but may suffer lost wages. This loss might be easily taken by some, but for others the loss would prove too great a financial burden. Are those who can afford to take time off work, and thus do so when ill, more morally responsible than those who do not? This would seem an unfair conclusion. How do we reconcile this situation? According to Harris and Holm, we look to

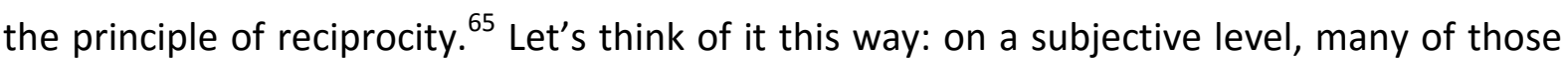
who are infected with a cold may not particularly mind working whilst ill. Yet, if they did go to work and infect others at the place of work, the result may be others becoming ill, who do take time off work. Ultimately, this may result in a loss in revenue or efficacy for their place of work. ${ }^{66}$ Therefore the benefit of one staying home when ill may not be a question of direct harm to those they may infect, but it may also benefit those they actually work for. Therefore, society has multiple reasons to maintain an interest in keeping individuals who are ill with an infectious disease, at home. That said, if society doesn't make it easy for individuals to stay at home when ill or inadvertently punishes them for doing so, isn't the society just as morally liable as the sick individual for any harm that may be caused by the individual not remaining at home when ill? I would argue that it is. It is this thinking that is the basis for the principle of reciprocity: balancing an individual's interests and responsibilities with that of the societies.

If the principle of reciprocity is a solution to the above conundrum, then how exactly do we understand that principle? According to the above argument, there appears both a practical and moral reason for the idea that we cannot reasonably expect the individual to bear all the burdens and responsibility of isolating themselves when they are ill. A society as a whole benefits in multiple ways when an individual who is infected with a communicable disease isolates themselves; in many cases more than the infected individual. Therefore, we can reasonably expect that an ethical and pragmatic society would facilitate the isolation of the individual and aid in lifting some of the burdens borne as a result of isolation. So here

\footnotetext{
${ }^{64}$ Harris \& Holm, Is There A Moral Obligation Not To Infect Others? pg. 1215.

${ }^{65}$ Ibid.

${ }^{66}$ Ibid.
} 
Joshua Mclvor, The Ethics of Infectious Disease Control.

we have the principle of reciprocity: when an individual is a vector for an infectious disease, they have an obligation to refrain from spreading the disease to others and in turn the wider society should take responsibility for some of the burdens of self-imposed isolation. In other words, it shouldn't be a particularly difficult choice for someone to decide to isolate themselves in the event they become a vector for an infectious disease, as the society should facilitate this course of action. Harris and Holm apply this principle specifically to the context of the workplace in their paper: "A strict duty to stay home from work when one is infected with the common cold or flu would create financial hardship for many people. It therefore follows from the reciprocity thesis that such a duty, if it were to be imposed by legislation or by the informal rules of a particular workplace, would have to be balanced by compensation for any loss. ${ }^{67}$ It is worth noting here that it would be prudent to outline a probabilistic threshold to determine the necessity of an infected individual's isolation (assuming the individual is physical fit and capable of work). If an infected individual carries an illness with a very low chance of infecting others, it may be more beneficial to both the individual and the employer to allow that individual to work. The main point here is that if the expectation is for the individual to stay at home so as to not infect others, this burden must be shared by society as well as the individual.

We can see this principle in action in countries like New Zealand, which legislate that employers must provide employees with a minimum number of days of sick leave each year, for which they suffer no loss of pay and should not be penalised for in any way. ${ }^{68}$ Though this fact, and indeed the principle of reciprocity generally, may seem a trivial or even common sense moral act, the willingness for a society to share the burdens of those infected with a communicable disease, is a fundamental step when it comes to the ethics of infectious disease control. I would argue that the principle of reciprocity is perhaps the first moral principle to consider when it comes to infectious disease control. Furthermore, more complex and advanced principles are a natural extension of this fundamental principle. The balancing of the individual's rights with the interests of the wider society while recognising the individual as a moral agent deserving of ethical treatment, is an important step in the ethics of infectious disease control. It was not too long ago that out of fear and a lack of

\footnotetext{
${ }^{67}$ Harris \& Holm, Is There A Moral Obligation Not To Infect Others? pg. 1215.

${ }^{68}$ These rights are outlined by Employment NZ at:

http://employment.govt.nz/infozone/businessessentials/basics/leave/sick-leave.asp.
} 
Joshua Mclvor, The Ethics of Infectious Disease Control.

understanding, those infected with a communicable disease were treated with no regard for their own wellbeing, hence the saying "treated like a leper".

An additional point Harris and Holm make is regarding the mode of transmission of an infectious disease. They ask "Does the mode of transmission matter?", when it comes to our obligations not to spread infectious diseases. ${ }^{69}$ Though the way it is framed in their particular paper is not too important for us here, the question of whether or not the mode of transmission of a disease affects our moral judgements, will become a major part of our ethical discussions in this paper. Harris and Holm ask whether certain acts, such as sexual intercourse, convey more moral responsibility to the recipient of an infectious disease, than say, airborne transmission. That is: does willingly taking part in high risk behaviours make you more morally culpable than simply catching an illness from someone in the work place? Ultimately Harris and Holm seem dubious that one could make such a moral claim "Therefore the mode of transmission itself cannot form any basis for a sound moral judgement." ${ }^{\prime 70}$ I agree with Harris and Holm here and am not interested in going into the details of the argument, as ultimately it will not impact our discussion that much. Furthermore, even if one argues that there is a certain degree of responsibility for both parties when it comes to high risk behaviours, the real question we are interested in here is of the duties of individuals not to infect others. It is a sound claim that even if there is some degree of responsibility one must take when engaging in high risk behaviours (such as unprotected sex); this doesn't lessen the duty of those who have an infectious disease to not willingly put others at risk of infection. Regardless of mode of transmission, an individual with an infectious disease must take personal responsibility when it comes to not infecting others. Harris and Holm conclude:

"The moral duty to behave responsibly and not knowingly put other people at risk is not a duty that is confined to HIV infection or to other life threatening diseases... It is, however, also a duty which we can expect people to discharge only if they live in a community that does not leave them with all the burdens involved in discharging this duty. The diseases we have discussed - the common cold and flu - are usually regarded as fairly trivial... If a duty not to communicate diseases can be established for diseases of this kind it will be a general duty, and not a duty limited to serious or life threatening diseases." ${ }^{71}$

\footnotetext{
${ }^{69}$ Harris \& Holm, Is There A Moral Obligation Not To Infect Others? pg. 1216.

${ }^{70}$ lbid, pg. 1217.

${ }^{71}$ Harris \& Holm, Is There A Moral Obligation Not To Infect Others? pg. 1217.
} 
Joshua Mclvor, The Ethics of Infectious Disease Control.

By examining Harris and Holm's paper, we have established well-formed arguments for the moral duty one has not to infect others, not only in case of serious diseases, but also in the case of usually trivial diseases. Furthermore and perhaps more importantly, we have established the importance of the principle of reciprocity, a key principle to keep in mind as we move forward.

\subsection{Broadening principles: transparency, proportionality, and harm.}

We now have what I consider to be the most fundamental principle of the ethics of infectious disease control in our moral arsenal: the principle of reciprocity. So how can we expand from here? Remember that in this paper we are interested with infectious disease control and, as we have learnt from our EVD case study, control often means isolation and quarantine. Not only are these means of control important during catastrophic outbreaks, but our day to day running of the healthcare system should include sensible measures of quarantine and isolation. Therefore, if we are to have a sensible moral approach to infectious disease control, we will need to consider ethical principles that aid us in such situations. Where can we go from the principle of reciprocity?

Ross Upshur, who has written on the "Ethics and Infectious Disease" for the WHO's website $^{72}$ has also provided a useful paper on the "Ethics of Quarantine". ${ }^{73}$ In regards to our question on quarantine and isolation, Upshur offers "4 principles that must be met in order

for public health to contemplate an autonomy-limiting strategy". ${ }^{74}$ Each principle, though an ethical point in itself, is tightly linked with the others, so we shouldn't try to understand these principles as unique strategies. Rather we should view them as intended to work together as a complete moral framework. Let us now look at these principles and see what they have to offer. The first principle Upshur mentions is the harm principle. This principle suggests that in order for forced quarantine or isolation to be considered (an autonomylimiting strategy); there must be a clear threat of harm to the wider population, if the disease were to go unchecked. For example, in the case of EVD there is a clear risk of further transmission and thus harm if infected individuals are not isolated. However in the case of say, anthrax, an infected individual poses no risk of further transmission to other individuals

\footnotetext{
${ }^{72}$ Upshur, Ethics and Infectious Disease.

${ }^{73}$ Upshur, The Ethics of Quarantine.

${ }^{74}$ Ibid, pg. 1.
} 
Joshua Mclvor, The Ethics of Infectious Disease Control.

and thus isolation or quarantine is unnecessary. The principle seems to make intuitive sense: if we are to limit someone's autonomy, a move that shouldn't be taken lightly, we must firmly believe they pose a threat to the wider society, otherwise the limitation of autonomy is unjustified. The implications of the harm principle are far reaching and can offer much to an ethical framework. The principle raises two major factors which would seem to affect whether we deem a particular case of forced isolation or quarantine ethically justified. First, if we accept the principle, then we seem to have to accept that the degree to which we could consider autonomy-limiting strategies would depend on the potential harm caused by the disease itself; specifically it's virulence. If a disease is highly lethal, then it is likely to be considered to do more 'harm' than if it isn't lethal at all. This will determine whether we consider it justified to forcibly quarantine or isolate an individual in a particular scenario. For example: if someone is infected with EVD, then due to the virulence of the disease we may consider the potential harm it causes to be much higher than if they are only infected with the common cold. Therefore, we may consider it morally justified to isolate or quarantine someone in the case of EVD but not in the case of the cold (although remember according to Harris and Holm we may still criticise someone if they don't selfisolate in the case of the common cold, assuming the principle of reciprocity is met). So virulence seems to play an important role when considering the harm principle, especially if we wish to quantify the harm.

The second factor that will play an important role when considering the harm principle will be the mode of transmission of the disease. When considering how much harm an unchecked disease could cause, we have to consider how that disease is spread. There is also a strong pragmatic element here. Consider a disease like influenza, which is spread through an infected person coughing or sneezing which sending droplets through the air or through the hands of an infected person coming into contact, directly or indirectly, with another individual. ${ }^{75}$ In other words, influenza is pretty easy to spread around and if a particularly lethal or damaging strain came about, we may conclude that the ease of transmission has the potential to infect many individuals if the disease is left unchecked; thus the harm principle is met. With the harm principle met, we have strong ethical and pragmatic reasons to consider forced isolation or quarantine of infected individuals. Now

\footnotetext{
${ }^{75}$ WHO factsheet, Influenza.
} 
Joshua Mclvor, The Ethics of Infectious Disease Control.

consider a disease like malaria. Malaria is a vector-borne disease, meaning it is spread exclusively via an animal host, not directly human to human. ${ }^{76}$ Due to the nature of the vector, mosquitos, individuals infected with the disease pose no threat of spreading the disease to those around them and thus isolation or quarantine is unnecessary. The potential harm caused by allowing an individual infected with the disease to continue unchecked, is only to them, not to the wider society. Isolation and quarantine will have no effect on instances of the disease in the population, but protection from the vector has had proven impact on the instances of the disease. ${ }^{77}$ So we can see that whether or not the harm principle is met will also depend on the mode of transmission of the disease.

The second of Upshur's principles is the principle of proportionality or least restrictive means. This principle "holds that public health authorities should use the least restrictive measures proportional to the goal of achieving disease control." ${ }^{78}$ Thus, before considering mandatory quarantine or isolation, we should consider voluntary isolation options. This principle has both pragmatic and moral components. Practically speaking, if the risk is deemed low enough then voluntary isolation could be sufficient and fewer resources will be expended in the carrying out of a mandatory isolation or quarantine order. Morally, we see a strong adherence to the principle of reciprocity and the principle of harm in this principle. As far as the principle of reciprocity is concerned; if an individual is to have some of their autonomy removed in order to benefit the wider society, the society should allow that to be done voluntarily where possible, removing the harm that may be caused by the very process of forced isolation or quarantine. In relation to the harm principle, the risk posed to the wider society of the disease going unchecked may be low enough that voluntary isolation may be all that is necessary, so forced isolation would be excessive. However it must be kept in mind that this principle, in relation to the harm principle, may also swing in the other direction. Some illnesses may be so harmful or contagious and pose such a threat to the wider society, that the risk posed by any potential noncompliance with voluntary quarantine is simply too much. In these cases, it may be determined that the least restrictive means has to be forced quarantine and isolation in order to curtail a potentially catastrophic disease from spreading.

\footnotetext{
${ }^{76}$ WHO, factsheet, Malaria.

${ }^{77}$ Such as mosquito nets: CDC, Insecticide-Treated Bed Nets.

${ }^{78}$ Upshur, The Ethics of Quarantine, pg. 2.
} 
Joshua Mclvor, The Ethics of Infectious Disease Control.

The third principle Upshur discusses is the principle of reciprocity, which we have already discussed and will skip over. The fourth and final principle Upshur discusses is the transparency principle. This principle is straightforward and is a key principle in many democracies, beyond simply infectious disease control. If some individual's autonomy is going to be curtailed in order to protect the wider society, the justification and methods of this curtailing must be made clear by the body in charge of carrying out the orders. People should have a good idea of exactly why they are being, say, quarantined and what will happen to them. Information and support needs to be provided throughout the process and we can once again see the principle of reciprocity creeping in here. Practically speaking, the dissemination of information may increase compliance, as individuals will understand their civic duties if well informed of them. Ethically speaking, transparency will lessen the element of the unknown and thus the unnecessary fear one may experience if they are ignorant as to why they are being isolated or quarantined. By knowing what is going to happen to them and why, individuals affected by an infectious disease will be more at ease, which benefits both them and the bodies in charge of controlling the disease.

From Upshur's paper, we now have a greater understanding of some important principles when it comes to infectious disease control, specifically in relation to the issue of quarantine and isolation. Though many other infectious disease control strategies exist and ethical questions arise from them (think back to the question of the use of experimental treatment during the EVD outbreak), quarantine and isolation are two of the most basic yet effective strategies. Upshur summarises:

"In summary, then, quarantine is a blunt instrument to use in the control of infectious diseases. However, in some circumstances it is one of the only possible means of responding to an infectious disease threat... However, public health professionals must continually update their information in order to refine the exposure criteria, so that people are not needlessly quarantined. Hence, communication between public health professionals and clinicians is crucial... Though many of these actions may be controversial, particularly when they begin to affect the livelihood of individuals, this is not an excuse for deviating from a control strategy. Transparency and communication are crucial in this regard." ${ }^{79}$

We saw the effectiveness of isolation and quarantine strategies in the EVD outbreak. Although there were certainly major gaps in the ethical justification of the strategies used

\footnotetext{
${ }^{79}$ Upshur, The Ethics of Quarantine, pg. 3.
} 
Joshua Mclvor, The Ethics of Infectious Disease Control.

during the EVD outbreak (think of the "cordon sanitaire" strategies, mention by Gostin et al., which left many without food), ${ }^{80}$ we can see that when battling a disease that we know little about or have no effective treatment for, quarantine and isolation may be our two biggest weapons in fighting the disease. Furthermore, Upshur makes it clear that infectious disease control must be a multilateral effort, with all parties constantly updating their information and disseminating it amongst the other parties. Strategies are going to be multitiered, from the health care professional on the ground, to the microbiologist working on treatment in the lab, to the epidemiologist studying the trends and patterns of the disease. A single tier or party working in discordance with the others will not effectively control the disease and may jeopardise the entire effort.

\subsection{Do the ethics of infectious disease control vary depending on disease?}

Now that we have a better understanding of some of the important principles regarding the ethics of quarantine, isolation, and infectious disease control more generally, we can turn back to the question that is the basis for this section of the paper: do the ethics of infectious disease control vary depending on the disease? Earlier I said we must be wary of generalising ethical conclusions that we have learned from the EVD outbreak. This applies to any disease outbreak; though we must analyse and learn from disease outbreaks, we mustn't be too hasty to assume the same strategies will work in every case. We must be flexible and not make assumptions that what worked in one case will necessary work in the next case. I am hoping that by now the reader is beginning to get a strong sense that the answer to the primary question of this section of the paper, is 'yes'. In this subsection I would like to apply the principles we have analysed so far to a few different diseases and see how our ethical conclusions may vary. I would like to note here that these principles are intertwined and often whether or not we adhere to one in a particular case will depend on whether we adhere to the others and in what way. All the principles relate to what is essentially a science: infectious disease control. As such they all depend on physical facts. One physical fact about a disease may affect our analysis of multiple ethical principles, this is exactly the point I hope to get across. It is therefore inevitable that whilst trying to pull apart some of these principles and examine them under different lights, I will not be able to

\footnotetext{
${ }^{80}$ Gostin et al., West Africa's Ebola Epidemic is Out of Control, but Never Had to Happen, pg. 3-4.
} 
Joshua Mclvor, The Ethics of Infectious Disease Control.

separate them perfectly and there will be overlap. So I will make my best attempt to 'carve nature at its joints'.

If Upshur is right, then we must adhere to four principles when considering any autonomy-limiting strategy. I think we have established thus far that we have pretty good reason to accept Upshur's argument, at the very least in the sense that some sort of principled moral guideline must be followed when restricting an individual's freedom, regardless of whether we agree with Upshur's principles specifically (I do and I think there is compelling reason to). Furthermore, I have already suggested that Upshur's principles may extend beyond just quarantine and isolation; to many ethics of infectious disease control issues. Think back to the question of the use of experimental treatment during the EVD outbreak. Some of the "conditions" that were deemed necessary to meet were principles like 'transparency'. We could no doubt gain some valuable insight if we applied the other four principles to the case as well.

Let's first examine a disease we have already discussed and is right down one end of the 'spectrum' when it comes to harm: the common cold. We have examined in some detail how to apply the principle of reciprocity to something like the common cold, so let's see how we fare when applying multiple principles to this disease. Let's first ask the question: would we ever be ethically justified in considering autonomy-limiting strategies with regards to the common cold? It seems unlikely. First, let's try to apply the harm principle to this case. What is the potential harm to the wider society if someone infected with the common cold is allowed to continue unchecked? The potential harm would be pretty minimal. The common cold is not lethal, does not require any medical treatment (other than to manage symptoms) and can normally be fought off by our immune system with relative ease. It poses no major threat to public health safety. Nonetheless it is an airborne disease and relatively contagious, therefore, as Harris and Holm pointed out, it poses a predictable risk of infection to those who come into proximity with an infected person, such as family, friends, and colleagues. Furthermore, individuals taking work off when sick can cost employers money, which is at least some form of harm. Maybe mandatory isolation is warranted? 
Joshua Mclvor, The Ethics of Infectious Disease Control.

Though the harm principle may be met at a minor level, there is still the principle of proportionality. Given the minimal amount harm the disease can cause if left unchecked, it would not only be a waste of resources but also unnecessarily harmful to the individual, if mandatory isolation measure were to be taken. Why not offer up the option of voluntary isolation? If individuals understand the potential harm infecting others with the common cold can cause, they may very well be motivated to isolate themselves willingly, out of a sense of duty. Are the risk that they wouldn't choose to self-isolate and the potential harm caused by such a decision high enough to warrant intervention? Again, it seems unlikely. But we have one final principle to fall back on, just in case we do believe the risk is high enough: the principle of reciprocity. As already discussed, if we remove some of the burdens of selfimposed isolation from the individual, they will be even further motivated to prevent the spread of the disease. Thus, if we provide the individual with an option such as paid sick leave from work, then we as a society have probably met our obligations under the principle of reciprocity. There will of course still be a remainder of people, who despite everything will still risk infecting others but we can again return to the harm principle; is the risk of harm to the greater society really significant at this point?

Finally, the principle of transparency: how do we meet it in the above scenario? Well, so long as individuals know of their entitlement to, say, sick leave and their right to not be persecuted for taking it, then this principle and our societal obligations under it seem to be met. In this scenario we have deemed autonomy-limiting strategies unethical and unnecessary, so there is no call for transparency with regards to these strategies, as they are not being used. So we can see how Upshur's four principles apply to a case of the common cold. What about more difficult cases? Furthermore, I would like the reader to keep in mind the question: what is it about the common cold that has led us to our conclusions in this section? Was it the low virulence of the disease, the way it can be treated, how it is spread, or perhaps a combination of these factors? How about the question of the use of experimental treatment in the case of the common cold? Would we arrive at the same conclusion as we did with EVD? These questions are going to help us in the creation of an infectious disease taxonomy that will be the centrepiece of the third section of this paper.

The next disease we are going to look at is TB. TB, like the common cold, is spread through the air. That said, TB has relatively low pathogenicity, the WHO suggesting "People 
Joshua Mclvor, The Ethics of Infectious Disease Control.

infected with TB bacteria have a lifetime risk of falling ill with TB of $10 \%{ }^{\prime \prime 1}$ However, individuals who may be infected yet not present with TB can still transmit the disease, thus we can imagine it may have a high inapparent infection rate, meaning many infected individuals would go undiagnosed. Finally, TB is much more virulent than the common cold, killing millions every year. ${ }^{82}$ At this point, we have introduced three major differences between TB and the common cold: pathogenicity, inapparent infection rate, and virulence. ${ }^{83}$ How will this affect our application of Upshur's principles and are some factors more significant than others? I will answer this question by comparing TB to the common cold and controlling for the various differences, thus allowing us to examine one physical factor at a time. First, let's look at the pathogenicity of TB. Imagine if TB was identical to the common cold in that it had very low virulence, so was relatively benign. Would the decreased pathogenicity affect our application of Upshur's principles in any major way? I would argue it largely doesn't. If we can establish pretty low harm potential for the common cold, then the lower pathogenicity of TB would only further decrease the harm potential. If this were the case then we can more or less say of TB what we have already said about the common cold. What of the increased inapparent infection rate? This is intimately linked to low pathogenicity. Once again, we need to imagine that TB was just as virulent as the common cold but infected many without presenting symptoms. Once again, controlling for virulence, TB seems to pose no greater threat than the common cold and thus Upshur's principles would seem to apply much the same. Isolation would still be recommended as a voluntary measure because, although the disease may not present itself in many individuals, its ability to nevertheless spread from those who are infected but show no symptoms would create an equal obligation of isolation (as the common cold) for those who actually do present with symptoms. No, it would seem as though the pathogenicity and inapparent infection rate are ultimately not hugely significant factors in determining the difference between TB and the common cold when it comes to applying Upshur's principles.

The most obvious significant difference between TB and the common cold seems to be the virulence of the diseases. Both are transmitted in the more or less the same manner,

\footnotetext{
${ }^{81}$ WHO, factsheet, Tuberculosis, pg. 1.

82 Ibid.

${ }^{83}$ We could further break down TB by its various strains, such as MDR-TB and XDR-TB (discussed in the Speaker case). The fact that these strains make the disease even more difficult to treat would only heighten the difference between TB and the common cold.
} 
Joshua Mclvor, The Ethics of Infectious Disease Control.

yet one frequently kills its host while the other doesn't. Therefore, if we are to apply the harm principle to TB in this case, we would find that the potential harm caused to a society by a TB infected individual continuing unchecked, is much higher than with the common cold. Indeed, this potential risk was highlighted by the Speaker incident. If Speaker had infected others during his travels we could reasonably expect that some of those infected may have eventually died, or infected others who subsequently died. The same could not be said if Speaker simply had the common cold. At the very least, the harm caused by the cost and length of TB treatment, is a significant difference between TB and the common cold. So we seem to have a stronger answer to the harm principle when it comes to TB.

What about the proportionality principle? We can see that in the case of Speaker, health officials did instruct Speaker to refrain from his travels, offering Speaker the option of voluntary isolation. This would have been the least restrictive means, yet Speaker travelled regardless. Due to the potential harm Speaker could have caused on his travels, the option of forced isolation should have been on the cards for the relevant health professionals to order at their discretion.

Finally, what about the principle of reciprocity? As we know, TB requires much more intensive treatment than the common cold. Therefore, there are strong pragmatic reasons to isolate individuals in a hospital or somewhere they can receive health care without running the risk of infecting others. What if they cannot afford healthcare? I would argue, that in the case of diseases like TB, society cannot expect individual's to be isolated and simply left to die. Under the principle of reciprocity, if one is to isolate themselves and limit their autonomy, then one can expect health care treatment in return. This treatment should also include food and shelter, and offer as much as is feasible in order for the individual to maintain a 'normal' life. As for the principle of transparency, there seems to be little difference between the common cold and TB, in the sense that transparency should be ubiquitous, it will simply be the content of the information made 'transparent' that changes. With a cold the individual should know they have a right to take sick leave, with TB the individual should know their rights as well, but also understand clearly that due to the increased social risk they may be required to be forcibly isolated. So it would seem that when it comes to TB, due to the higher virulence and more demanding medical treatment of the disease, there is more ethical grounding to support the forced isolation and quarantine 
Joshua Mclvor, The Ethics of Infectious Disease Control.

of an individual (so long as all of Upshur's principles are met). Though it is difficult to carve nature at its joints, it would seem as though the most significant difference between the common cold and TB, when it comes to our ethical conclusions, is the virulence of the diseases. Though other factors certainly play a role, the virulence of the diseases makes the biggest difference.

Moving forward, let us now continue to control for virulence, to see if we can arrive at any other morally significant differences between infectious diseases. I would like to look at the mode of transmission. Let's take a look at bubonic plague. The bubonic plague is arguably more virulent than $\mathrm{TB}$, but we only need to accept that both have the ability to cause death and are very serious illnesses. Furthermore, some types of plague, like pneumonic, can spread via the air from person to person, so we need to focus on bubonic plague, which is spread via the bite of fleas. ${ }^{84}$ So for our purposes, the significant difference between TB and bubonic plague is the mode of transmission: TB is spread through the air and plague is spread through fleas. Let us also say that most other factors are more or less equal, simply for the thought experiment at hand. We found that there could be good cause for the isolation of individuals with TB; will we find the same for individuals infected with bubonic plague? There are strong moral and practical reasons to suggest not. Ethically speaking, the harm principle appears not to be met in the case of bubonic plague. An individual infected with bubonic plague poses no consistent risk to those around them and a lack of treatment is only going to cause harm to the infected person. The difference in the human to human spread between the diseases is that spread is done directly with TB but done via fleas with bubonic plague. Therefore, physical isolation is of no use when it comes to bubonic plague, as infected fleas can still transmit the disease. However, awareness and targeting of the potentially infected fleas is of great use. Without the harm principle being met for bubonic plague (in order to justify isolation or quarantine), we cannot expect the other principles to fall into place either. That said, this does not eliminate all ethical quandaries for the case of bubonic plague. Small animals potentially infected with plague, maybe a necessary food in some parts of the world, could be outlawed from being eaten or sold during outbreaks, perhaps even permanently. This would apply the harm principle in a different manner; not in relation to the question of quarantine and isolation, but in relation

${ }^{84}$ WHO, factsheet, Plague, pg. 2. 
Joshua Mclvor, The Ethics of Infectious Disease Control.

to the question of prohibition. Allowing individuals to buy and sell potentially infected small animals may allow further spread of the disease to continue, thus sufficiently fulfilling the harm principle. Only in this case the harm principle may justify the banning of certain forms of meat, or may even call for the culling of certain animals. Here we have a perfect example of something I said earlier: Upshur's principles don't simply apply to the matter of quarantine and isolation. We can now see different ethical outcomes between TB and the bubonic plague, which result from a difference in mode of transmission. If we see different ethical outcomes due to mode of transmission in the case above, then we have no reason to doubt it will occur in relation to varying other diseases, all with varying modes of transmission.

In this subsection I hope to have shown strong evidence that the ethics of infectious disease control vary greatly depending on the biological features of the disease itself. Furthermore, certain biological features of a disease may impact our conclusions, upon applying Upshur's principles, more than other features. Though this is hardly a definitive outcome, I have already suggested that virulence and mode of transmission may have a big role to play when it comes to our ethical judgements of infectious disease control. By looking at several different diseases, we have gained some practical understanding of exactly how virulence and mode of transmission may affect our ethical judgements when it comes to infectious disease control. However, I would like to suggest that there is something further missing here. In the first section of the paper I said EVD is a "stage three" disease as identified by Wolfe, Dunavan, and Diamond. ${ }^{85}$ Ultimately, this fact had a large influence on the WHO's decision to consider the use of experimental treatment ethically justified during the outbreak (though they don't directly refer to it as I do). Recall the quote: "The only way of obtaining evidence on the safety and efficacy of any intervention in Ebola virus disease is during an outbreak, because identified sporadic disease is very rare." ${ }^{86}$ This fact, that we can only study EVD interventions in humans during an outbreak, is a direct result of the disease being a stage three disease. Furthermore, we can wonder whether the same conclusion would be reached if the disease wasn't a stage three disease; I don't think it would have been.

\footnotetext{
${ }^{85}$ Wolfe et al., Origins of major human infectious diseases.

${ }^{86}$ WHO, Ethical considerations for use of unregistered interventions for Ebola viral disease, pg. 4.
} 
Joshua Mclvor, The Ethics of Infectious Disease Control.

\subsection{The missing link: the evolutionary stages of diseases.}

The title of this subsection may be a bit histrionic, but I believe it fitting for this point in the paper. So far we have examined some of the ethical difficulties that the 2014 EVD outbreak provided and analysed the discussion that surrounded these difficulties. We have looked in detail at some of the founding principles that underlie the ethics of infectious disease control and tested these principles in different situations. Finally, we have begun to realise that the biological features of infectious diseases have a profound impact on what we deem to be ethical courses of action when it comes to controlling the infectious diseases. We have a couple biological feature candidates that look promising so far: virulence and mode of transmission. We have examined how these two factors may impact our application of Upshur's ethical principles. All of that said there is a subtle, yet important, gap which was illustrated in the case of the use of experimental treatment during the EVD outbreak. Though I have attempted to lead to this conclusion as naturally as possible, I will have to take a leap here and simply offer the theory up. That theory is outlined by Wolfe, Dunavan, and Diamond in their paper "Origins of major human infectious diseases" ${ }^{87}$ In this subsection I will outline the theory and suggest why it is of ethical significance. ${ }^{88}$

The paper examines 25 major infectious diseases, analyses and categorises them into the five evolutionary stages, and distinguishes them into "Old World" and "New World" diseases, as well as "Temperate" and "Tropical" diseases. ${ }^{89}$ Though the "Old World" vs. "New World" and "Temperate" vs. "Tropical" distinctions will be of no major significance for our purposes, the five evolutionary stages will be. Wolfe et al. selected their 25 major infectious diseases with the aim being "...to select well-defined diseases causing the highest mortality and/or morbidity and hence of the highest historical and evolutionary significance." 90 The evolutionary stages mark the "transformation of an animal pathogen into a specialized pathogen for humans." ${ }^{91}$ Furthermore, "There is no inevitable progression of microbes from Stage 1 to Stage 5: at each stage many microbes remain stuck, and the

\footnotetext{
${ }^{87}$ Wolfe et al., Origins of major human infectious diseases.

${ }^{88}$ The paper covers much and contains 59 pages of supplemental notes. Inevitably, I will only be able to cover a very small portion of this; focusing on what I deem most relevant to our discussion. I would encourage the reader to read the paper to get a better understanding of the evolutionary stages and their significance.

${ }^{89}$ Wolfe et al., Origins of major human infectious diseases, pg. 279.

${ }^{90}$ Ibid.

${ }^{91} \mathrm{lbid}, \mathrm{pg} .280$.
} 
Joshua Mclvor, The Ethics of Infectious Disease Control.

agents of nearly half of the 25 important diseases we selected for analysis have not reached Stage 5." ${ }^{\prime 2}$ I have summarised what the five stages are below:

- Stage 1: The disease is found in nonhuman animals, but has not been found in humans in natural conditions.

- Stage 2: The disease is found in nonhuman animals and has been transmitted from animals to humans, but not between humans.

- Stage 3: The disease is found in nonhuman animals and has been transmitted from animals to humans and between humans. However, the disease only undergoes a few cycles of 'secondary' transmission. ${ }^{93}$ Therefore Stage 3 disease outbreaks tend to die out naturally in human populations.

- Stage 4: Same as Stage 3, except the disease can sustain long sequences of secondary transmission, thus remaining in the human population for much longer than stage 3 diseases (sometimes they remain in the human population permanently). ${ }^{94}$

- Stage 5: The disease is exclusive to humans.

Wolfe et al. categorise the 25 major infectious diseases into each evolutionary stage in their supplemental notes, but I will provide examples in a table in section three of this paper. The stages are visualised nicely in the figure on the next page, taken from Wolfe et al.'s paper:

\footnotetext{
92 Wolfe et al., Origins of major human infectious diseases, pg. 280.

93 "Secondary" transmission is human to human transmission after initial 'primary' transmission of animal to human.

${ }^{94}$ Wolfe et al. subdivide this stage into three further stages. However, these three sub-stages are not of major significance to us and may only, needlessly, complicated the ethics further.
} 
Stage

Transmission to humans

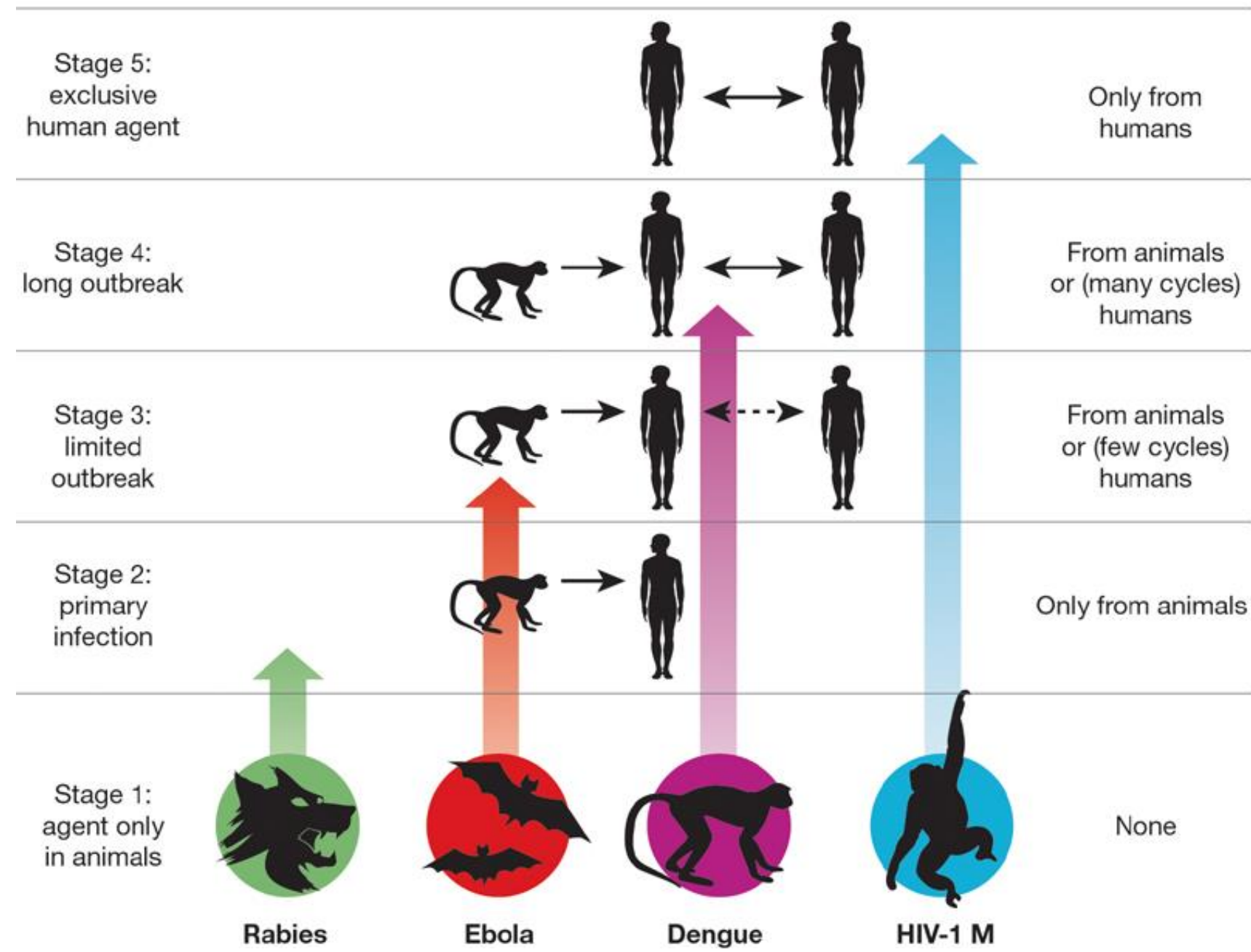

Figure 1 | Illustration of the five stages of the evolution of pathogens from exclusive to non-human animals, to exclusive to humans. The four agents depicted have reached different stages in the process, ranging from rabies (still only acquired directly from animals) to HIV-1 (now transmitted purely between humans). ${ }^{95}$

At this stage the reader should have a good enough understanding of Wolfe et al.'s theory of evolutionary stages of infectious diseases. Reading the article itself would of course further aid this understaning. Now we can look at the ethical implications of the evolutionary stages. Stage 1 diseases have not yet been transmitted from animals to humans, so the ethical implications of such diseases are limited, at least as far as our discussion here goes. Of course one important point must be made, which Wolfe et al. suggest: by monitoring diseases found in animals, especially in areas where animals come into close contact with humans, we can develop an "early warning system" so that we don't

${ }^{95}$ Wolfe et al., Origins of major human infectious diseases, pg. 281. 
Joshua Mclvor, The Ethics of Infectious Disease Control.

blindsided by novel infectious diseases. Wolfe et al. elaborate "Monitoring of people, animals, and animal die-offs will serve as an early warning system for disease emergence, while also providing a unique archive of pathogens infecting humans and the animals to which we are exposed." 96 The monitoring of all diseases, not just those found in humans, will inevitable improve our preparation and understanding of diseases that may pose a human threat sometime in the future. So concerns surrounding stage 1 diseases may, strictly speaking, be more pragmatic than ethical.

Stage 2 diseases can be transmitted from animals to humans but not between humans, therefore there will be no question of quarantine or isolation here despite the virulence of some of these diseases (e.g. anthrax). This example makes it pretty clear how the evolutionary stage of an infectious disease will play a big role in how we judge ethical actions. Previously, we noted that highly virulent diseases usually fulfil the principle of harm better than low virulence diseases. Yet, this analysis is affected even further by the evolutionary stage of the disease: there can be no question of harm caused to the wider society if the infected individual was left unchecked, as they physically cannot spread the disease to other humans. Once again, ethical questions of the type we are interested in here will be few and far between when considering Stage 2 diseases. There will be concerns similar to that raised by Stage 1 diseases such as: the monitoring of diseases in animals in order to increase our understanding of, and preparedness for, such diseases could be advisable. In addition, an increased understanding of how the disease presents itself in humans may aid in our understanding of the disease's potential to begin secondary transmission between humans. This is one of the key areas Wolfe et al. consider us to have little understanding of: "Less well understood... is the transition from Stage 2 to Stage 3 and 4..." ${ }^{97}$ There may also be ethical questions as to whether or not the prohibition, temporary or permanent, of certain animal products in certain areas may be warranted. Though this may affect peoples' livelihood, the risk of transmission of the disease from particular animal products to humans, may be significant enough during particular outbreaks of the disease. Though many animal products can be treated, this scenario isn't entirely unlikely.

\footnotetext{
${ }^{96}$ Wolfe et al., Origins of major human infectious diseases, pg. 283.

${ }^{97}$ lbid, pg. 279.
} 
Joshua Mclvor, The Ethics of Infectious Disease Control.

Stage 3 diseases can maintain a few cycles of secondary transmission between humans. These diseases, like EVD, offer unique problems of their own. First of all, due to their ability to be transmitted between humans, the issue of quarantine and isolation arises. Whether or not the measures are justified will depend on the particular disease, as well as factors like virulence and mode of transmission. Second, as with EVD, these diseases often only undergo a few cycles in humans before disappearing back to their reservoir host. ${ }^{98} 99$ This means there is often a limited window of opportunity to study the disease and potential treatments, in humans. As the WHO found, this may strengthen the claim that the use of experimental treatments is justified because it is not often the opportunity to learn about the efficacy of such treatments in humans arises. ${ }^{100} \mathrm{~A}$ final issue is how we view these diseases in broad terms. Of course, when outbreaks occur we need to react to them and offer help to those affected by the diseases. Beyond that however, we need seriously to consider the destabilising threat of such diseases. When we think about hugely destabilising infectious diseases, ones that have had a massive impact around the globe, Stage 3 diseases do not always come to mind. This is directly due to their nature of not being able to maintain multiple cycles of human to human transmission. Ultimately whether or not this fact is of much significance is something to be decided and thought about by policy makers, but it is still worthwhile mentioning.

Stage 4 diseases maintain long and sustained cycles of secondary transmission between humans and pose many difficulties. Stage 4 diseases, like influenza, can maintain transmission between humans for greatly extended periods of time and as such, have the potential to cause significant damage to civilian infrastructure. Of course, as with EVD, there will be questions of isolation and quarantine when it comes to Stage 4 diseases. However, due to the fact that Stage 4 diseases don't tend to disappear back into the reservoir host after a few cycles, more permanent strategies will be preferable when dealing with Stage 4 diseases. By this I mean development of effective treatment is very important. Although there may be a call for the use of experimental treatment during particularly bad outbreaks,

\footnotetext{
${ }^{98} \mathrm{~A}$ "reservoir host" is a place the disease persists for long periods of time, usually inside the body of an animal although sometimes in environmental bodies such as water. In animals, the reservoir host is usually unaffected by the disease, or at least not killed by it, as this would make it's persistence in the particular species of animal unviable.

${ }^{99}$ Obviously it is not as if the disease physically "retreats" back to its reservoir host: it is always there. Simply, due to its inability to maintain more than a few cycles in humans, it can disappear from humans altogether, yet continue to persist in the reservoir host.

${ }^{100}$ WHO, Ethical considerations for use of unregistered interventions for Ebola viral disease, pg. 4.
} 
Joshua Mclvor, The Ethics of Infectious Disease Control.

due to the persistence of these diseases in the human population, there is not a substantial urgency required to rush the development process, as opposed to the 2014 EVD outbreak. There isn't as much of a limited window of opportunity. It may even be unethical to use experimental treatment during the normal levels of occurrence of Stage 4 diseases due to the potential harm they may cause (depending on the circumstances). Because of their ability to be 'reseeded' into the human population from the reservoir host and their ability spread between humans for extended periods of time, Stage 4 diseases are arguably some of the worst we know of. Thus, ethical considerations will be many and may change quite dramatically on a case by case basis.

Stage 5 diseases are found only in humans. Due to this fact, we have had some success in eliminating these diseases from the population, as they have no reservoir host to 'retreat' to. Once again there will be questions of isolation and quarantine with Stage 5 diseases. These diseases pose another interesting ethical question: should vaccination against such diseases be mandatory ${ }^{101}$ The eradication of Smallpox in 1980 due to an immunization campaign led by the WHO highlights the major benefits that can arise from widespread vaccination against Stage 5 diseases. ${ }^{102}$ Unlike all the diseases covered previously, Stage 5 diseases don't have animal or environmental reservoir hosts, therefore sufficient prevention of spread between humans can see the disease eradicated for good, without a fear of re-entry into the population. This fact raises the question: when a safe and accessible vaccine has been created for such diseases, should the vaccine be mandatory? If the vaccine was made accessible to all individuals who are viable candidates, it is hard to see what motivation individuals would have to not vaccinate, thus mandatory vaccination seems unnecessary. However, the recent anti-vaccination movement and subsequent increase in preventable illnesses has shown that people can be easily corrupted by a poor understanding of science. ${ }^{103}$ Furthermore, if vaccination only benefited the person receiving the vaccine, the question may be null and void. That is not the case though; widespread vaccination creates 'herd-immunity', protecting those who cannot be vaccinated as well as those that can. Additionally, if the vaccination doesn't have a $100 \%$ success rate then the

\footnotetext{
${ }^{101}$ This question probably applies to all diseases to a varying extent. That said, the potential benefits seem to increase as disease become less reliant on animal to human transmission. Once they are exclusively human diseases, the benefits of successful vaccination campaigns are massive.

${ }^{102}$ WHO discussion on this at: http://www.who.int/csr/disease/smallpox/en/.

${ }^{103}$ Sifferlin, 4 Diseases Making a Comeback Thanks to Anti-Vaxxers.
} 
Joshua Mclvor, The Ethics of Infectious Disease Control.

more individuals that are vaccinated, the less chance there is of the disease spreading from someone for whom the vaccine hasn't worked, to another individual for whom the vaccine hasn't worked. Ultimately, I would argue mandatory vaccination will more often than not be ethically justified if in a pathogen had sufficient capability to widely affect a large population. Admittedly this is relatively vague, which is a reflection of the issues at hand: they are not clear cut. An example of a scenario in which mandatory vaccination was ethically justified might be a strain of diphtheria that is highly virulent, and has a high infectivity/pathogenicity, but is not currently being well controlled, leading to many deaths. If there was an effective vaccine available, mandatory vaccination would be justified in such a situation (although I think people would be very willing to vaccinate in this scenario). So we can see that, as well as many of the questions raised by diseases of other evolutionary stages, Stage 5 diseases offer unique ethical problems of their own.

In this subsection we have examined Wolfe et al.'s theory of evolutionary stages of diseases. We have looked at each stage and suggested some of the unique ethical dilemmas posed by diseases in each stage. I hope to have made a strong case for the idea that, like virulence and mode of transmission, the evolutionary stage of a disease has a big impact on how we deal with some of the ethical dilemmas posed by different infectious diseases.

\subsection{Summary}

So far in this paper we have achieved several things. First, by looking at the case of the 2014 EVD outbreak in West Africa, we have obtained a real world sense of some of the ethical challenges the issue of infectious disease control poses. Additionally, we now have an in depth understanding of a real and significant example of an infectious disease outbreak, which we can draw upon throughout this thesis. Second, we have taken a groundup approach to the ethics of infectious disease control. Starting with the paper by Harris and Holm and moving on to Upshur's paper, we unravelled some of the ethical grounding for several core principles of the ethics of infectious disease control. We examined how the application of these principles is altered dramatically depending on some key biological features of diseases. By looking at various infectious diseases and how they differed from one another, we came to understand how dramatically the ethical problems and outcomes 
Joshua Mclvor, The Ethics of Infectious Disease Control.

changed based upon features like virulence and mode of transmission. Finally, as an important last piece of the puzzle, we looked at Wolfe et al.'s paper on the evolutionary stages of major infectious diseases. The theory offered another important biological feature of infectious diseases, which dramatically impacted how we deal with the various, and different, ethical issues raised by different diseases. By this point in the paper we should ultimately have a strong sense of how the virulence, mode of transmission, and evolutionary stage of a disease can have massive implications on how we apply well founded ethical principles to that disease, in a certain scenario. Now, we are ready to put what has been learnt so far into practice and develop a type of 'infectious disease taxonomy' that will provide the basis for an ethical framework to be used in infectious disease control. 
Joshua Mclvor, The Ethics of Infectious Disease Control.

\section{Section Three: A Disease Taxonomy Ethical and Policy}

\section{Implications.}

\subsection{Introduction.}

Now that we have a strong basis for the claim that biological features of a disease have a major impact on our ethical and pragmatic approach to the disease, we can utilise this information. In this section of the paper I will present a disease taxonomy drawing upon the virulence, mode of transmission, and evolutionary stage of the disease. I will start by discussing the current approach taken to infectious disease control in New Zealand (NZ) and discuss some of the potential shortcomings of the approach. Then I will present the disease taxonomy itself and briefly describe it. From this I will outline the major categories (or 'cells') the disease taxonomy entails and discuss some of the ethical implications for each category. I will conclude by suggesting how such a taxonomy could be used to guide the ethics infectious disease control policy. It will be made clear that my intention is not to suggest that current infectious disease control policy is fundamentally flawed or that my disease taxonomy is better; I have neither the resources nor the intricate understanding of the bureaucratic processes to do so. I am a moral philosopher. What I hope to make clear is that my disease taxonomy is an ethical suggestion, intended to offer a potential route of further investigation that could lead to an improvement in infectious disease control policy. The taxonomy in its current form is not intended to replace current policy, but perhaps supplement it, or provide an incentive to further research the potential use of such a taxonomy.

A final note is that my intention in this paper is by no means to provide a comprehensive answer to the multitude of ethical questions surrounding infectious disease control. So far the paper raises as many questions as answers - this is my intention (although I still hope to have found some solid ethical conclusions, grounded in reasoned argument). I have tried to illustrate the intricate relationship between ethics and pragmatics and I have indeed suggested that any serious ethical theory will accommodate practical concerns: ethicists should not conduct their work locked in ivory towers. Therefore, I respect those whose job it is to arrive at conclusions to many of the ethical questions raised throughout this paper and though I am willing to offer up suggestions, I will acknowledge their expertise 
Joshua Mclvor, The Ethics of Infectious Disease Control.

when it comes to answering such questions. My taxonomy does not look to answer all the ethical questions it poses, but rather looks to direct further ethical discussion on the issues raised in a clear and precise manner. That is to say, the intention of the taxonomy is to hasten the ability of whoever may be interested, to ask and thus answer the most pertinent and relevant questions in relation to the ethics of infectious disease control. It is a road map to aid in the understanding of the landscape of the ethics of infectious disease control; a tool to be used to clarify and hopefully simplify the ethics of infectious disease control. The work of the paper so far has been to provide the practical and theoretical groundwork for the development of such a tool.

\subsection{Current approach to infectious disease control in New Zealand.}

The management of infectious disease control strategies in New Zealand, particularly in the event of a potential outbreak, is covered by five pieces of key legislation:

1) The Health Act 1956.

2) Health (Infectious and Notifiable Diseases) Regulations 1966.

3) Health (Quarantine) Regulations 1983.

4) Bill of Rights Act 1990.

5) The Epidemic Preparedness Act 2006. ${ }^{104}$

Each piece of legislation contributes crucial legal backing in a particular area, in order to aid in the overall process of infectious disease control. Together, the statutes achieve several goals. First, they give the government the necessary statutory power to effectively respond to potential epidemics. For example, the Epidemic Preparedness Act 2006 outlines the special powers conveyed to the prime minister in the event of an infectious disease outbreak. ${ }^{105}$ Second, the statutes work together to outline the rights of those who may be impacted by infectious disease control measures, especially in the case of quarantine and isolation. For example, the Health (Infectious and Notifiable Diseases) Regulations 1966 outline the limitations of healthcare professionals' power to isolate individuals, including the length of isolation. Finally, the statutes together provide a clear set of expectations and limitations in advance of any potential infectious disease outbreak, thus the processes

\footnotetext{
${ }^{104}$ Links to all of the Acts in the references.

${ }^{105}$ Epidemic Preparedness Act 2006, Section 5.
} 
Joshua Mclvor, The Ethics of Infectious Disease Control.

involved in controlling the outbreak are transparent. As we have already discussed, the principle of transparency is a key principle to adhere to when utilising infectious disease control strategies, both for its ethical and pragmatic implications.

In addition to the legislation that provides the legal backing for both individual rights and governmental powers during various stages of infectious disease control, there are also a couple of important, non-legislation documents when it comes to infectious disease control in NZ. These documents are:

1) The National Ethics Advisory Committee: Ethical Values for a Pandemic. ${ }^{106}$

2) Ministry of Health: Influenza Pandemic Plan. ${ }^{107}$

What these documents cover is pretty indicative from their titles. They provide information and guidelines to those who will be on the frontline when responding to any epidemic such as medical staff, emergency services, community workers, and so on. They outline measures through which such individuals and the wider community can prepare for, respond to, and recover from an epidemic, whilst maintaining high ethical standards. The core purpose of these documents is outlined well in the Influenza Pandemic Plan:

"It is important to note that ethical considerations are broader than the legislation suggests, and that the law is silent on many issues raised in pandemic planning. In addition, the law is often slow to follow moral change in the community, so older legislation may not necessarily reflect a community's current ethical values." ${ }^{108}$

In this quote we can already begin to see the necessity for ethical frameworks, such as that which will be provided in this paper, to plan for issues that aren't covered by the law. Moving forward, it is important to keep in mind that the Ministry of Health's "Influenza Pandemic Plan" is exactly that: a plan in the eventuality of an influenza pandemic. Of course this is a sensible plan to have written, but as we have already seen we cannot necessarily expect the ethical guidelines outlined in this plan to apply to different infectious diseases. The issue of key biological differences between infectious diseases is not addressed in The National Ethics Advisory Committee's Ethical Values for a Pandemic either, although the example scenarios provide a great ethical basis for general behaviours that should be encouraged during an infectious disease outbreak. The only writing I could find that

\footnotetext{
${ }^{106}$ National Ethics Advisory Committee, Getting Through Together: Ethical values for a pandemic.

${ }^{107}$ Ministry of Health, New Zealand Influenza Pandemic Plan: A framework for action.

${ }^{108}$ Ibid, pg. 15.
} 
Joshua Mclvor, The Ethics of Infectious Disease Control.

highlighted the ethical importance of physical differences between infectious diseases, was the Epidemic Preparedness Act 2006 in which incubation times of diseases, and thus the appropriate isolation periods, was outlined for a decent number of major infectious diseases. ${ }^{109}$ Though this 'list' based approach is certainly very useful and most of the major diseases we might expect to occur in NZ are covered, there may be difficulties when dealing with novel pathogens and ethical questions beyond simply isolation and quarantine for the diseases listed. I hope my disease taxonomy could help strengthen, in some way, these measures that are already in place. Below I have outlined the process, based upon some of the documents I have already outlined, that would occur in the event of an infectious disease outbreak. The colours signify the severity of that particular stage in terms of threat to the NZ public.

The Process of Infectious Disease Control in NZ:

\section{Preparation:}

Utilization of frameworks such as those set up in the Influenza Pandemic Plan. Monitoring of diseases and communication within the health sector is used to stay ahead of any potential epidemic. Incidences of notifiable diseases listed in Schedule 2 of the Health Act 1956 are reported to the medical officer of health, so any abnormalities can be quickly reported. Monitoring of disease spread overseas is utilised to predict a potential pandemic, and WHO advice is adhered to.

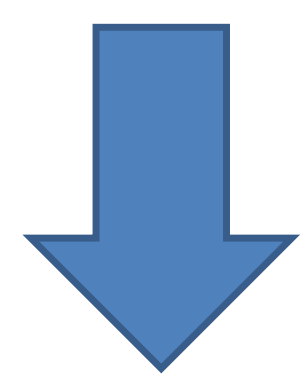

${ }^{109}$ Epidemic Preparedness Act 2006, Schedule 2. 


\section{Overseas Epidemic Alert:}

An epidemic is occurring overseas. Monitoring is very important and travel restriction may be necessary (1). If the outbreak is occurring in two or more countries in one WHO region, efforts to keep the disease out of the country must be taken, as suggested by the Influenza Pandemic Plan. This would require heavier than normal monitoring of activities at border ports. Local health bodies need to begin preparations for a potential epidemic by doing things such as: increasing protective equipment, creating more hospital ICU space, and taking staff through the procedures.

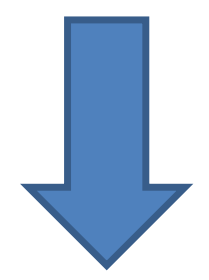

\section{First Cases in New Zealand:}

Upon arrival of the first cases in New Zealand, isolation of the disease and further spread prevention must be prioritised. Per the Health (Infectious and Notifiable Diseases) Regulations 1966, the Environmental Health Officers shall visit and assess the premises of the infectious person (S7a) and report his finding to the Medical Officer (S7b). He shall repeatedly visit and monitor the situation to ensure appropriate measures are being taken to prevent further disease spread (2) (S7). He will report to and be guided by the Medical Officer of Health $(\mathrm{S} 7 \mathrm{~m})$ and may require occupants to remain in isolation for the period of time stated in Schedule 2 of the Regulations (3). Discretion is given to the Medical Officer of Health as to the terms of isolation and medical examinations to be carried out.

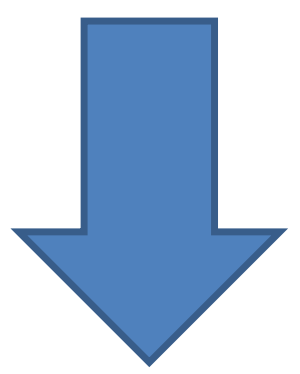




\section{Increasing Number of Cases in New Zealand:}

With the agreement of the Minister of Health, the Prime Minister may issue an epidemic notice as per the Epidemic Preparedness Act 2006 (S5(1)). The notice is to be issued if the Prime Minister believes the epidemic will disrupt NZ society. The notice will be instruction for Parliament to meet immediately (S6) and ensure government has sufficient power to coordinate a cohesive response to the threat, which will ultimately be led by the Ministry of Health. It will be at the discretion of Medical Officers of Health to quarantine and isolate individuals for the length of the incubation period outlined in Schedule 2 of the Health (Infectious and Notifiable Diseases) Regulations 1966(4). Upholding common ethical values, outlined in "Ethical Values in a Pandemic" will be of great importance to ensure continued social cohesion and wellbeing.

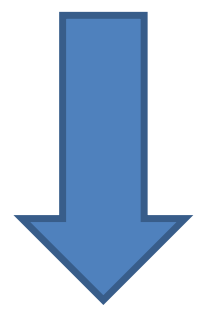

\section{Recovery Phase:}

Procedures to recover from a major epidemic are outlined in the "Influenza Pandemic Plan". Steps are taken to prevent a resurgence of the disease, whilst planning is made to prepare for such resurgence. Restoration of normal services is important and schools may begin to reopen. Depending on the nature of the illness, a vaccine may be rolled out so risk of resurgence will decline as the population's immunity increases (5). Services are created to aid in the recovery of those businesses, schools, and social groups who were hit worst by the epidemic. As strain on emergency services eases, hospitals can revert to normal operations.

I have numbered and highlighted in red five major ethical problems that arise form the current process of infectious disease control outlined above. First is the issue of travel restrictions. In the early stages of a potential pandemic, when outbreaks have occurred overseas; travel restrictions in order to prevent the spread of the disease to NZ may be justified. However, there is no reason why Upshur's principles couldn't apply to situation. I have already stated I think the principles are applicable to more than just the issue of isolation and quarantine. Though it may not seem as much of a violation of individual rights 
Joshua Mclvor, The Ethics of Infectious Disease Control.

as, say, quarantine or isolation; limiting one's ability to travel is not something that should be done lightly. There should be clear evidence that the harm principle is met: that unchecked travel of certain individuals does pose a major threat to the wider society. Whether or not this harm principle will be met, will depend largely on the factors of the disease itself. For example, a malaria outbreak overseas does not warrant the prevention of persons potentially infected with malaria from travelling, but it may in the case of influenza. Whatever the case, the harm principle must clearly be met and the same goes for Upshur's other principles. In the case of reciprocity: as much as is possible authorities should strive to offer alternative travel arrangements or recompense. Individuals caught out and unable to return to work, should not be allowed to lose pay or be otherwise persecuted by their place of employment. In the case of proportionality: if individuals can be advised to voluntarily refrain from travelling instead of being physically prevented from travelling, then this option should be considered first. Finally, in terms of transparency, authorities should make information regarding the current and predicted state of travel into, within, and out of NZ readily available and accessible.

The second ethical issue I have highlighted is the duties of the Environmental Officer. It is important to note that the duties of the Environmental Officer shouldn't be limited to monitoring individuals to ensure further spread is prevented, but should also include aiding the wellbeing of the infected individuals. This fits well with the principle of reciprocity; if we are forcibly isolating infected individuals, in their home or elsewhere, we should ensure these individuals are well looked after. This should be an important part of the Environmental Officer's duties, or at least some other health care professional who comes into contact with infected individuals. The third ethical issue follows closely on from the second; if individuals are in isolation for a predetermined period of time, all of Upshur's principles must be met. There must be a clear expectation of harm if the individual wasn't isolated, the process and reason for isolation must be made clear to the individual, they should receive necessary medical treatment, and they mustn't be punished in their place of employment. These points should all be intuitive conclusions by this stage in the paper.

The fourth ethical issue highlighted is more or less the same as the third, except we must now consider the same issues for the case of quarantine. We must strive to once again meet all of Upshur's principles. In many cases, because quarantine deals with individuals 
Joshua Mclvor, The Ethics of Infectious Disease Control.

who are not confirmed to be infected but are suspected likely being infected, Upshur's principles must be even more firmly met: the evidence to support them must be substantive. In regards to the harm principle, we must have sufficient reason to believe quarantined individuals are of a high enough risk to cause substantial potential harm. To uphold the least restrictive means principles, quarantined individuals must, wherever possible, be advised to isolate themselves of their own volition before they are forcibly isolated. The principle of reciprocity can be met in a number of ways. Individuals must once again not be punished at their place of work because of their quarantine and individuals should not be put in a situation where they are at high risk of contracting the disease from other quarantined individuals. Furthermore, if they are confirmed to be infected they should receive adequate medical treatment, or not be expected to be isolated. As is always the case, there should be transparency about the entire process and individuals should be informed of their rights.

The fifth and final ethical issue I have highlighted is whether or not vaccines should be made mandatory after such an outbreak. According to the Health (Infectious and Notifiable Diseases) Regulations 1966 ordering mandatory vaccination against smallpox was within the power of the authorities. Should we expect the same while recovering from any future outbreak? It would seem unlikely that anyone would refuse a free vaccination for a disease that has just caused an outbreak within NZ, but we must still consider the possibility. Once again, the answer would depend on whether Upshur's principles are met by this course of action. The harm principle, like in the case of smallpox, may be clearly met, if the risk of unvaccinated individuals continuing to spread the disease is substantial. Reciprocity would include support for anyone who experienced adverse side effects from the vaccine, as well as time off work if needs be. The least restrictive means would suggest offering the vaccines for free at community centres before starting to monitor and force individuals to take their vaccine. Finally, transparency once again entails information be provided justifying the whole process.

These five ethical issues I have outlined appear to be resolved by the relatively straightforward process of applying Upshur's principles in each case. Though this is certainly a good start, I did not go into detail on how the biological features of the diseases would substantially affect our ethical conclusions in each of these cases. I think this provides strong 
Joshua Mclvor, The Ethics of Infectious Disease Control.

evidence for the need of a disease taxonomy, such as the one I will suggest, to be incorporated into the process of infectious disease control. Though it may be that some of the ethical issues I have raised are addressed elsewhere in legislation or documents, I have not managed to find definitive answers on these issues. Furthermore I haven't discovered any suggestion that the biological features of diseases plays an important role in the ethics of infectious disease control. Though I do not doubt the relevant healthcare professionals' ability to adapt to a variety of infectious diseases and scenarios, the ethical issues I have raised show a gap, in the policy surrounding infectious disease control in NZ. I hope my disease taxonomy may offer means by which to plug this gap or at least lead to further discussions that would do so.

\subsection{The infectious disease taxonomy}

On the next page I have inserted my infectious disease taxonomy table. The colour coding creates groups of diseases that I think will have similar answers to certain ethical principles. The more dark red the colour, the more of a threat to global communities those diseases tend to be. I have also not included Stage 1 diseases, as those diseases have not yet been transmitted to humans, therefore there will not be any major ethical issues of interest for the purposes of this paper. I mentioned last section the imperative for research and observation to be done on these Stage 1 diseases in order to increase preparedness for potential outbreaks, but that is more of a pragmatic issue than an ethical one. 


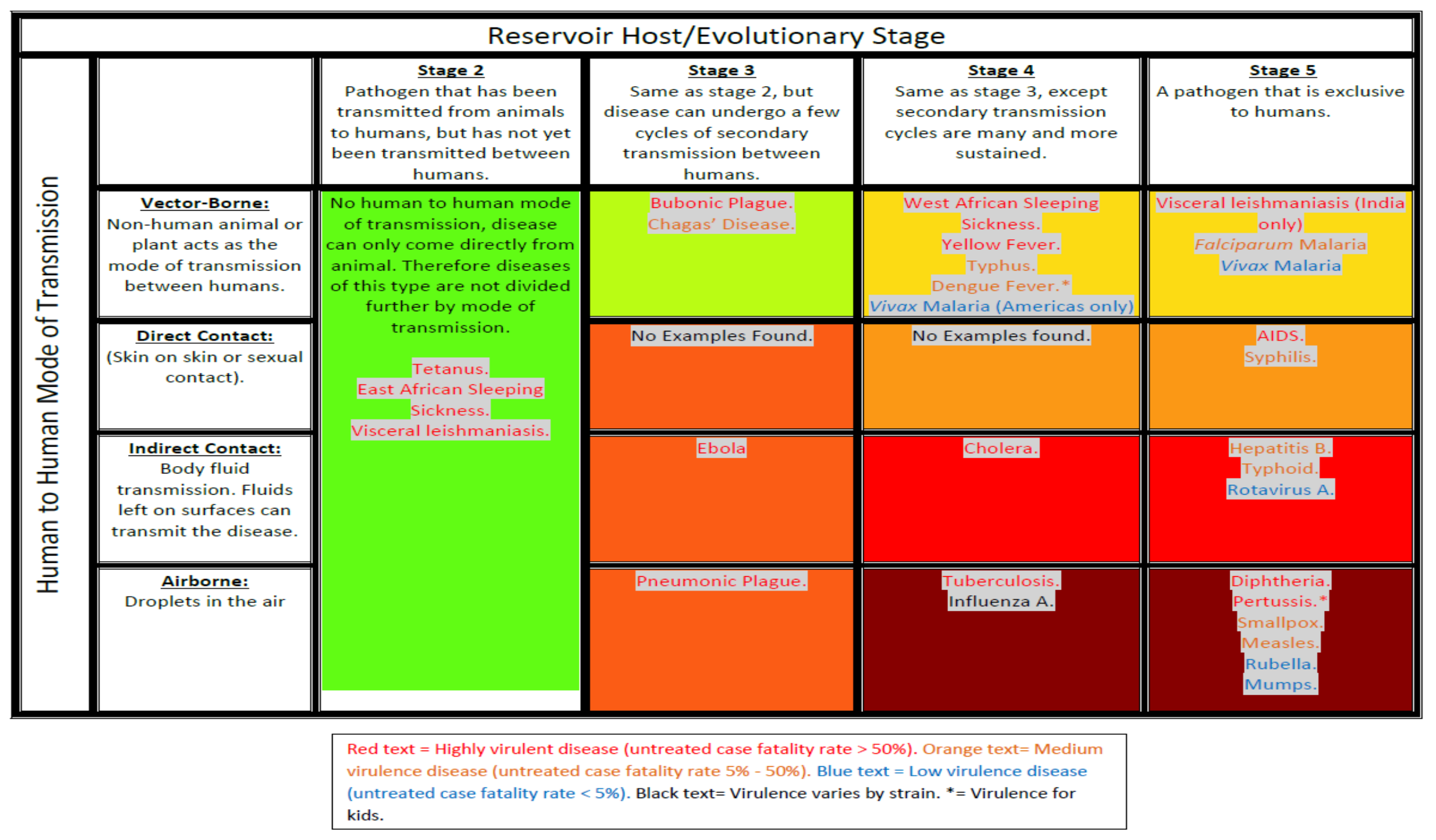

Figure 2 | Infectious Disease Taxonomy Table. All figures have been taken from Wolfe et al.: Supplementary notes, pg. 4 (link in the references). 
Joshua Mclvor, The Ethics of Infectious Disease Control.

Before continuing, I would like to make a couple notes about the table. First, the colouring of the cells does not necessarily indicate morally significant differences in the diseases. As stated earlier, the colouring is a general indication of the threat posed to the global community by the disease. It is certainly debatable how we define "threat" as many individuals, who should by no means be neglected, routinely die of the less 'threatening' diseases. That said, here I intend 'threat' to mean diseases that have the capacity to cause widespread damage to civilian infrastructure, the way that EVD did during the 2014 outbreak. The colours are not meant to represent precise borders but are more meant to represent a general theoretical threat potential of diseases. Obviously, even diseases that are in the more red zones will not be as damaging if they have low virulence. Second, there are a couple cells which contain no examples. This is not because none exist, but because I haven't thus far been able to confirm any diseases that fit into these categories. The evolutionary stage of a disease is not a commonly used categorisation tool for infectious diseases. Furthermore, the line between indirect and direct contact as modes of transmission, can blur. Diseases that can be spread through indirect contact have the propensity to be spread through direct contact as well, though not always. This means diseases like EVD, could actually fit into both categories, and thus fill both cells.

So, in the table we can see infectious diseases divided by the three major biological features we have already discussed: virulence, mode of transmission, and evolutionary stage. Thus, in each cell will be diseases that are the same evolutionary stage and have the same mode of transmission. This intersection generates unique outcomes to varying ethical problems between the cells. We have discussed some of these outcomes already, in section two. In addition to the factors utilised in the table, we must also remember that there are several other key factors that may affect the outcome to various ethical problems, some of which we have mention already. Below is a list of some additional factors that are worth considering when discussing the ethics of infectious disease control, though may not have as large an impact as the factors used to make the taxonomy table.

1) Pathogenicity/Infectivity: The more likely a pathogen is to cause (potentially) severe symptoms, the more precautionary we need to be when dealing with those who are ill. This will strengthen the claim of the harm principle being met for any particular illness: the more infective a disease is and the more likely it is to cause illness after 
Joshua Mclvor, The Ethics of Infectious Disease Control.

infection, the greater the risk an infected individual poses to the wider society. This will likely increase the ethical justification of cases of isolation and quarantine.

2) Inapparent infection rate: If a disease has a high inapparent infection rate (infectious people don't always present symptoms), this can be hugely problematic for epidemic control. Depending on the other features of the disease, this may increase the need for quarantine: there is an increased potential for those who have come in to contact with an infected purpose to not be aware that they have contracted the disease and are spreading it. Once again justified by the harm principle, quarantine may be required in order to test individuals who are at risk of having contracted the disease and confirm the cases.

3) Immunogenicity: Whether or not a disease will produce an immune response, ultimately preventing future infection, can have a huge effect on how we control the disease. This may increase the call to push for vaccination development and it allows individuals who survived infection and are no longer contagious to assist with those who are ill, without risk of reinfection. In an extremely bad situation, such as that depicted in the film Contagion (2011), immunity could be a way of dividing the population in order to better control the illness: those who are now immune can carry out civic duties. ${ }^{110}$

4) Point when contagious: Knowing at what point during an infection someone is contagious can be of great use in disease control. If someone is only contagious once symptomatic, as with EVD, then there may be less of a need for quarantine, as nonsymptomatic individuals will not be contagious (though may still need to be monitored). Furthermore, if someone is contagious well before or after they are symptomatic, more caution and quarantining, may be warranted.

Ultimately, the taxonomy table and the additional factors all boil down to information. The more we understand about various diseases and their biological factors, the better we are able to deal with them physically and ethically. We can then better argue that particular courses of action for particular diseases fulfil Upshur's principles, or that they don't. Of course it could be said that much of this is already known about the 25 major diseases that Wolfe et al. examined and that make up a decent portion of the list of

${ }^{110}$ Contagion, dir. S. Soderbergh, 2011. 
Joshua Mclvor, The Ethics of Infectious Disease Control.

infectious diseases in the Health (Infectious and Notifiable Diseases) Regulations 1966. Furthermore, if we don't know this information, then the taxonomy table seems to be useless. This is a valid concern, but I have a couple responses to it.

First, although the current legislative approaches that utilise lists of diseases undoubtedly provide much in the way of understanding for the diseases included in the list, they do not get at the heart of what is causing us to arrive at the ethical conclusions that we do. This makes the list-based approach inflexible; struggling to deal with novel threats. David Fidler, Lawrence Gostin, and Howard Markel outline this frustration in relation to the Speaker incident: "The Speaker case exposed problems with federal isolation and quarantine authorities. For example, federal powers apply to a specific list of diseases, thus depriving the federal government of flexibility when responding to novel threats. The listing approach requires the president, for each new threat, to make the disease quarantinable through executive order, which is what transpired when SARS and fears of pandemic influenza emerged." ${ }^{111}$ I argue that whilst comprehensive information of diseases is still needed when utilising my taxonomy table, it is more adaptive and quicker to deal with novel pathogens than simply having a list of known disease and what to do about them. In the U.S. each new disease has to be added to a list in order to exercise federal power. In NZ, this seems to be similar, with the lengths of isolation and quarantine determined on a disease by disease basis. ${ }^{112}$ The result is that novel pathogens would need to be added to such a list before substantial and effective action can be taken to mitigate them. By utilising the taxonomy table, or something like it, once a few biological features of an infectious disease were known, there is already a classification category in place to deal with ethical and pragmatic concerns.

The second issue ties in with the first; a list based approach is not streamlined. We essentially wind up with as many disease categories as there are diseases, which in the case of NZ is at least 26 diseases. ${ }^{113}$ Under my disease taxonomy table, we only have 13 categories which will not change even with the addition of novel infections (assuming the nature of infectious diseases doesn't change). This allows for a much more reactive and

\footnotetext{
${ }^{111}$ Fidler et al., Through the Quarantine Looking Glass: Drug-Resistant Tuberculosis and Public Health Governance, Law, and Ethics, pg. 621.

${ }^{112}$ Health (Infectious and Notifiable Diseases) Regulations 1966, Schedule 2.

${ }^{113}$ Ibid.
} 
Joshua Mclvor, The Ethics of Infectious Disease Control.

streamlined approach to infectious disease control. By focusing on the ethically significant physical differences between diseases, we can easily infer whether courses of actions deemed justifiable for one illness will pertain to another illness, instead of having to develop guidelines for each disease individually. This said, the best approach would probably combine both the list based approach and the disease taxonomy table. List based approaches have the advantage of providing in depth information that is relevant to each disease. The disease taxonomy is slipstreamed and offers more flexibility, increasing the ability to adapt to novel infections, whilst not putting all infectious diseases under one category of 'quarantinable'. Combining the two approaches may provide the best strategy for infectious disease control.

\subsection{An ethical framework for the infectious disease taxonomy.}

Based upon what we understand about the ethics of infectious disease control, what general ethical conclusions can we draw about each of the 13 cells of the infectious disease taxonomy table? I will go through each cell and outline some of the major points that we may consider ethically relevant for the diseases that fall into that category. I will try to focus on the ethical points that make each cell unique, not what makes them the same as other cells. Therefore, a bit of common sense and inference of certain points between cells may be necessary. I will start with the Stage 2 infections and move on from top left to bottom right of the remaining cells.

\section{Stage 2 Infectious Diseases}

Stage 2 illnesses are transmitted directly from animals to humans so the mode of transmission between humans is irrelevant. Questions of isolation and quarantine will be irrelevant as there is no human to human spread. Some of the major ethical issues surrounding such diseases may arise from animal control and animal product control. Though irrelevant for a disease like tetanus, which is found in soil, diseases like visceral leishmaniasis, which is found in dogs and rodents, may call for the possibility of culling groups of animals or preventing the trading of animals and animal products. Though first line protection from the animals that spread such diseases is probably the most ethical means of preventing the disease from spreading, outbreaks may become so bad that more extreme measures are required. If it is found that a population of animals poses a threat to 
Joshua Mclvor, The Ethics of Infectious Disease Control.

a society, then the harm principle will be met for courses of action like the culling of the animals. The same may be argued for the prevention of trading in animal products, though the reciprocity principle would require adequate recompense for those who may lose sources of income as a result of the ban.

\section{Stage 3, Vector-Borne Infectious Diseases}

Stage 3, vector-borne infectious diseases are transmitted from animals to humans and between humans via an intermediary vector, such as a mosquito or flea. Due to the nature of spread by vectors, questions of isolation and quarantine are unlikely to arise. Once again there will be the issue of animal and animal product control. Additionally, the question of experimental treatment may arise. Like EVD, these diseases only undergo a few cycles of secondary transmission between humans, therefore the opportunity to test treatment interventions may only arise during outbreaks. However, a point to keep in mind is that with diseases that are not vector-borne, the benefit of something like vaccination is two-fold: the individual is protected and they are also removed from the pool of potential vectors for the diseases, thus likely saving others from the disease. This double sided benefit may not hold when the mode of transmission is, say, a flea. If a flea were to come into contact with an infected person and thus carry the disease, even if the infected person then receives treatment and is no longer susceptible to the disease, the flea can continue to infect others with the disease. Though widespread vaccination may ultimately lessen the pool of susceptible individuals so greatly that the flea will no longer have a viable target, this process will most likely occur slower than when a human is the vector for transmission. Therefore, the potential benefits from using experimental treatment may not be as big as with other categories of diseases and the risks associated with experimental treatment may outweigh the benefits. Due to this, investment in protection from the vector itself may be the safer and more ethical option.

\section{Stage 4, Vector-Borne Infectious Diseases}

Stage 4, vector-borne infectious diseases are also spread via vectors such as mosquitos or fleas, except they can maintain extended cycles of human to human transmission. This fact will may lessen the efficacy of animal control strategies such as culls, as the disease can continue to transmit between humans for extended periods of time. 
Joshua Mclvor, The Ethics of Infectious Disease Control.

Thus, the potential benefits of such measures are lessened compared to the harms caused to the animals and those who rely on them. This will in some cases make such courses of action unethical. Furthermore, due to the persistence of these diseases in the human population, our opportunities to study the effects of potential treatment interventions will be greater than just during outbreaks. Therefore, the imperative to use experimental treatments is going to be lessened, except in the case where outbreaks are exceptionally bad.

\section{Stage 5, Vector-Borne Infectious Diseases}

Stage 5, vector-borne infectious diseases will require similar ethical considerations to all vector borne illnesses. However, due to the lack of an animal or environmental reservoir, these illnesses can theoretically be removed from the human population through effective treatment or prevention. Therefore, the question of mandatory treatment comes to mind: if treatment can prevent the spread of the illness, then enough treatment may be able to eradicate the disease. That said, we have already highlighted the difficulties of controlling disease when they are vector-borne: isolation and quarantine isn't effective. Treatment, if it produces an immunogenic response, may very well be effective in lessening susceptible individuals to a critically low level. However, treatment is sometimes expensive and time consuming, and a better strategy may be to enforce disease prevention measures in high risk areas. The mandatory use of mosquito nets in certain areas may dramatically impact the incidences of malaria, assuming of course they are provided to those who can't afford them. This is the strategy employed by the WHO, with great effect: "Vector control is the main way to prevent and reduce malaria transmission. If coverage of vector control interventions within a specific area is high enough, then a measure of protection will be conferred across the community."114 They report that "Between 2000 and 2015, malaria incidence (the rate of new cases) fell by $37 \%$ globally. In that same period, malaria death rates fell by $60 \%$ globally among all age groups, and by $65 \%$ among children under $5 .{ }^{115}$ Cleary the vector control methods are working, thus there is solid evidence that mandatory vector control interventions would meet Upshur's principles.

\footnotetext{
${ }^{114}$ WHO factsheet, Malaria.

115 Ibid.
} 
Joshua Mclvor, The Ethics of Infectious Disease Control.

\section{Stage 3, Direct Contact Infectious Diseases}

Stage 3, direct contact infectious diseases undergo few cycles of human to human transmission and are transmitted via direct (skin on skin or sexual) contact between individuals. Isolation and quarantine, though theoretically effective, are unlikely to meet the harm principle for these diseases. Individuals with these diseases usually pose low risk to the wider society due to the mode of transmission. According to the principles of least restrictive means, self-regulation is normally sufficient for controlling the diseases. In the same way you cannot get HIV from someone through holding their hand or kissing them, diseases that require direct contact are not easily spread outside of intimate contact with the individual and will not be caught in passing. Due to this fact, offering experimental treatment to stem the spread of the disease is also unlikely to be warranted, as simple practices like safe sex can prevent the spread of these illnesses. Furthermore, stage 3 diseases only undergo a few cycles of human to human transmission before dying out, so the major threat of these diseases may arguably be the animals from whence they came. This may call for animal and animal product control. Due to the mode of transmission requiring the animal to also make direct contact with the human however, awareness of safe animal control practices may satisfy the least restrictive means principles before any more extreme measures would be considered.

\section{Stage 4, Direct Contact Infectious Diseases}

Stage 4, direct contact infectious diseases will encounter many of the same problems posed by their stage 3 counterparts. The key difference is going to be stage 4 diseases' ability to persist in the human population for longer periods of time. Isolation and quarantine may be ineffective and focusing on the animal origins of the disease may not be enough to prevent the spread of the illness. Monitoring and tracking the disease may be the best option, as well as public health campaigns encouraging the safe practices required to prevent disease spread. The interesting ethical questions that relate to these diseases will relate to the section of Harris and Holm's paper that covers modes of transmission. ${ }^{116}$ Whether or not we view individuals who contract these diseases as a result of engaging in high risk behaviours as equally morally culpable will greatly

${ }^{116}$ Harris \& Holm, Is There A Moral Obligation Not To Infect Others? Pg. 1216. 
Joshua Mclvor, The Ethics of Infectious Disease Control.

influence how we deal with the disease in terms of societal norms. As I said earlier, any claim that those who engage in high risk behaviours must take moral responsibility for their actions will not negate the duty of those who are infected to not infect others. Furthermore, bestowing moral culpability on to those who engage in high risk behaviours and thus contract the disease from such behaviours, poses an intuitive conflict with our views of health care workers who frequently engage in high risk behaviour through their work. Ultimately, this cell of infectious diseases may not have much different to say in terms of ethics and policy, but more about ethics and social values/views.

\section{Stage 5, Direct Contact Infectious Diseases}

There are some examples of stage 5, direct contact infectious diseases in our table, unlike the prior two classes of diseases. Sexually transmitted infections (STIs) like AIDS are common stage 5 direct contact infectious diseases. Once again, questions of isolation and quarantine are unlikely to yield much. These diseases are exclusive to humans and so the imperative arises to try and eradicate them from the population. A point I haven't mentioned thus far in the paper, is that the viability of achieving eradication can differ greatly between developed and developing countries. This is due to a number of social, economic, and political reasons, making it very difficult to implement worldwide disease eradication strategies. For example, HIV treatment has come a long way since it was first discovered and those living in developed countries who contract HIV an receive diagnosis early enough, can expect to relatively normal lives. Yet "Sub-Saharan Africa is the most affected region, with 25.8 [24.0-28.7] million people living with HIV in 2014. Also sub-Saharan Africa accounts for almost $70 \%$ of the global total of new HIV infections." ${ }^{117}$ Nonetheless, efforts to eradicate the disease will always pose ethical questions. Mandatory STI testing of sex workers, free or cheap contraception, and criminal charges for those who knowingly infect others with HIV are some of the ethical dilemmas that surround these diseases perhaps more than any other cell. I would maintain that so long as Upshur's principles are met, most, if not all, of these actions are ethically justified.

${ }^{117}$ WHO factsheet, HIV/AIDS. 
Joshua Mclvor, The Ethics of Infectious Disease Control.

\section{Stage 3, Indirect Contact Diseases}

Stage 3, indirect contact disease are the first of the cells we've examined to seriously raise questions of isolation and quarantine. Due to their ability to spread through bodily fluids, which can often remain on surfaces and still be infectious, these diseases call for isolation of those who are sick. The degree to which the harm principle will be met will depend largely on the virulence of the disease. As we discussed in section two of the paper, diseases with very low virulence, such as the common cold, don't pose a major threat to society. However, diseases like EVD, which falls into this cell of the taxonomy table, do pose a threat to society - if left unchecked in individuals. Therefore, given Upshur's principles are met; isolation and quarantine will often be warranted with these diseases. Furthermore, as we have already discovered with EVD, understanding and treating these diseases may call for us to make exceptions to our normal operating procedures and allow the use of experimental treatment during outbreaks. These diseases can and do spread rapidly and wreak destruction on civilian infrastructure, so the imperative to control, learn from, and prepare for these diseases during and after their relatively short lived excursions into the human population, is substantial.

\section{Stage 4, Indirect Contact Infectious Diseases}

Once again, the major issue with stage 4, indirect contact infectious disease when compared to their stage 3 counterparts, is their ability to sustain prolonged cycles of human to human transmission. This means we can expect these diseases to be a persistent and prolonged issue within populations. While isolation and quarantine will certainly be justified for those diseases that are virulent or harmful enough, the use of experimental treatments shouldn't be utilised under the same pretext as the EVD outbreak. We will often have an opportunity to study these diseases in humans outside of outbreaks. This also means long-term strategies will need to be incorporated to keep the incidences of such diseases low. For example, cholera can be transmitted through incorrect hygiene practices so the issue of whether or not, say, restaurants that infect their customers with the disease should face prosecution, arises (assuming the required hygiene practices are readily available). I would argue that this may be necessary. Failing to adhere to hygiene practice in a professional capacity seriously compromises the safety 
Joshua Mclvor, The Ethics of Infectious Disease Control.

of the general public. That said, under reciprocity the government should make it easy for everyone to be able to meet hygiene standards.

\section{Stage 5, Indirect Contact Infectious Diseases}

Stage 5 indirect contact infectious diseases, like their stage 3 and 4 counterparts, are often easily spread around. Quarantine and isolation will be necessary when the risk of further transmission, and virulence, is high. The question of mandatory treatment and prevention arises, as with other stage 5 diseases. Another ethical question that arises is: should those infected with say, hepatitis $B$, not be able to work in certain sectors? It is a difficult question and not one to be brushed over. Hepatitis $B$ “...can survive outside the body for at least 7 days. During this time, the virus can still cause infection if it enters the body of a person who is not protected by the vaccine." ${ }^{118}$ This clearly poses a huge risk of further transmission, in some sectors more than others. Or perhaps the vaccine itself should be made mandatory. This is probably the more equitable solution. The risk of transmission and the possibility of eradicating the disease provide strong reason to support the ethics of mandatory vaccination in these cases, assuming Upshur's principles are met.

\section{Stage 3, Airborne Infectious Diseases}

Airborne infectious diseases are (usually) more contagious than indirect contact infectious diseases. Therefore the harm principle is usually even easier to meet for airborne infectious disease that are highly virulent, than indirect contact infectious diseases. Due to the likelihood of these diseases to spread with ease, quarantine measures will be more ethically justified than any of the disease cells examined so far. Individuals who may have come close to someone on, say, a plane, are much more likely to have contracted the illness than if the disease was spread through indirect contact. If the disease is harmful enough, quarantining those on the plane with a potentially infected individual would be warranted. Furthermore, as with EVD, diseases in this cell will likely flare up for relatively short periods of time. Therefore the ability to study the disease in humans during outbreaks is often the only opportunity. EVD would have been

${ }^{118}$ WHO factsheet, Hepatitis B. 
Joshua Mclvor, The Ethics of Infectious Disease Control.

much worse had it been an airborne disease and as such the use of experimental treatment would have been even more ethically justified, due to the harm principle being met much easier. All of this said, in theory these diseases shouldn't persist for long in the population and so many of the goals will be short term: control the disease during outbreaks.

\section{Stage 4, Airborne Infectious Diseases}

Stage 4, airborne infectious diseases pose an even bigger threat than their stage 3 counterparts. They can sustain much longer cycles in the population and can be "reseeded" into the population from their animal reservoirs. These are arguably some of the most threatening diseases to society. A great example of a disease in this category is influenza A. Influenza A has caused panic in the past and potential unforeseen strains of the disease will likely cause us problems in the future. When the disease is highly virulent, it would be hard to see isolation and quarantine measures not being ethically justified for these diseases. Furthermore, I would argue there is a strong basis for mandatory vaccination when possible. Even seasonal influenza - the 'flu'- experienced every year, is damaging enough to potentially warrant mandatory vaccination. The misconception of influenza as simply a strong cold, means the motivation to vaccinate is often minimal in much of the population. Most fit and healthy individuals will not suffer any major consequences of seasonal influenza, but clearly the purpose of the vaccination is not necessarily to protect these individuals, but to minimise the potential vectors of the disease and thus protect those who may not be candidates for the vaccine. With diseases like influenza, herd immunity is imperative. Due to having reservoir hosts, these diseases are unlikely to go away in the future and so in addition to isolation and quarantine, other control methods like mandatory vaccination, should be considered.

\section{Stage 5, Airborne Infectious Diseases}

We now arrive at our last cell in the disease taxonomy table. It is the cell with the most of the 25 major infectious diseases outline by Wolfe et al. These diseases are exclusive to humans and are transmitted through the air and many of these diseases are vaccinated against in childhood. Once again there is a theoretical potential to eradicate these diseases and many have decreased dramatically in developed countries due to 
Joshua Mclvor, The Ethics of Infectious Disease Control.

widespread vaccination. Isolation and quarantine is crucial in many cases here and as we saw with the Speaker incident, the risks to society by allowing infected individuals to continue unchecked are large. The case for mandatory vaccination when available is stronger for these diseases compared to stage 4 diseases, due to the potential to eradicate the diseases. Recall the smallpox vaccine was able to be forcibly administered under the Health (Infectious and Notifiable Diseases) Regulations $1966 .{ }^{119}$

That concludes this subsection of the thesis. After outlining some of the major ethical issues posed by diseases of each cell of the infectious disease taxonomy table, we should have a good idea of some of the considerations that need to be made for the various diseases. Furthermore, by focusing on the disease categories, or cells, I have highlighted the importance of focusing on the biological features of infectious diseases when it comes to ethical control strategies. I did not bring the additional biological features, outline in the previous subsection, into the equation here, because the reader should have a good, intuitive idea of how these would strengthen or weaken certain ethical claims in each infectious disease category. Ultimately, this section was intended to outline some of the ethical conclusions that arise from each infectious disease category.

\subsection{Using the framework: Zika virus and measles.}

There has been a lot of recent media attention surrounding Zika virus, particularly in relation to increased cases of children born with microcephaly in Brazil, thought to be a result of pregnant women contracting Zika virus. ${ }^{120}$ What would our ethical framework and disease taxonomy have to say about containment methods for the virus? Zika virus is spread through the bite of the Aedes mosquito (although there have been two cases of potential sexual transmission) and it causes flu like symptoms. Zika virus is lowly virulent and treatment is similar to that of a cold or flu: plenty of rest and fluids. I haven't been able to find the evolutionary stage of the disease, but it has been detected in rhesus monkeys and can spread between humans, so it must be either a stage three or four disease. ${ }^{121}$ The

\footnotetext{
${ }^{119}$ Health (Infectious and Notifiable Diseases) Regulations 1966, Section 20.

${ }^{120}$ WHO factsheet, Zika virus.

${ }^{121}$ Ibid.
} 
Joshua Mclvor, The Ethics of Infectious Disease Control.

biggest issue of Zika virus seems to be that it may cause microcephaly in children if the mother is infected with the disease during pregnancy. ${ }^{122}$

What can we conclude about Zika virus, using the ethical guidelines outlined above? It is likely a stage three or four disease of low virulence, which is spread through mosquitos and is possibly sexually transmitted. Quarantine and isolation are not going to be ethically justified, as due to the mode of transmission, this will be ineffective. Experimental treatment is also unlikely to be ethically justified, as the disease is of a very low virulence and the potential side-effects of experimental drugs are of a greater cost than the potential benefits. However so long as conditions are met, such as those that were required for the 2014 EVD outbreak, an argument could be made that if some promising experimental drugs looked to prevent microcephaly occurring in new-borns upon their mothers use of the drug, then such experimental treatment could be ethically justified. There would have to be a very suitable experimental treatment option, with very low risk. That said, such measures may not meet the principle of proportionality. A better, more affordable and less risky option, might be to make disease testing available for those who wish to get pregnant, as well as offering widespread contraception. Furthermore, as I stated in the previous subsection, vector control and prevention will always be the most direct means of controlling vector-borne disease (once again ensuring any adverse effects are accounted for under the principle of reciprocity and least restrictive means). This is the strategy advised by the WHO:

"Mosquitoes and their breeding sites pose a significant risk factor for Zika virus infection. Prevention and control relies on reducing mosquitoes through source reduction (removal and modification of breeding sites) and reducing contact between mosquitoes and people.

This can be done by using insect repellent regularly; wearing clothes (preferably light-coloured) that cover as much of the body as possible; using physical barriers such as window screens, closed doors and windows; and if needed, additional personal protection, such as sleeping under mosquito nets during the day." ${ }^{\prime 123}$

Ultimately, improved family planning and vector control strategies will likely prove a more ethical, less risky alternative to utilising experimental treatment. If the disease is sexually transmitted, there will be strong moral obligations for those that know themselves to be infected to not engage in unprotected sex, without informing their partners first. Failure to

\footnotetext{
${ }^{122}$ WHO factsheet, Zika virus.

123 Ibid, pg. 2.
} 
Joshua Mclvor, The Ethics of Infectious Disease Control.

do so may be considered a form of assault or harm and is at least unethical and at most justified in being prosecutable.

Another incident that has been in the media recently is that of a person infected with measles risking the health of others by ignoring instructions to isolate themselves, instead choosing to roam a busy casino. ${ }^{124}$ As I said in section 3.2 , the medical officer of health, who in this case was Dr. Richard Hoskins from Auckland, does have the legal authority to order people into quarantine, but "Usually, a heavy-handed approach wasn't required." ${ }^{125}$ What can we say about the incident? Measles is a stage five, airborne disease, with a medium virulence level. ${ }^{126}$ Due to this relative severity, and the fact that a safe and effective vaccine is available, it is a disease that we should very much want to, and can, eradicate. Undoubtedly this individual, who knowingly risked the health of many people by ignoring medical advice, has committed an unethical act. Should the medical officer have ordered the individual into mandatory quarantine? I don't think so. If it is correct that a heavy-handed usually isn't required, ${ }^{127}$ then it must be the case that most individuals willingly follow their medical professional's advice. Furthermore, most individuals in developed countries, like NZ, are immunized against measles at birth and "In 2014, about $85 \%$ of the world's children received 1 dose of measles vaccine by their first birthday through routine health services, up from $73 \%$ in $2000 .{ }^{\prime 128}$ Clearly we are moving towards herd immunity and it is unlikely the individual in this case would have come into contact with many non-immunised individuals. So in this case, advising selfimposed isolation would have fulfilled both the least restrictive means principle, the principle of proportionality, and the principle of harm. What could be said of the circumstance, is that there is ethical grounding for medical authorities to have the ability to fine those, like the individual in question, who ignore medical advice and put others at risk by exposing them to an infectious disease. We can compare this to how we needn't put speed inhibitors in cars in order to stop people speeding, that'd be excessive, and the risk of being fined would be a more proportional deterrent.

\footnotetext{
${ }^{124}$ New Zealand Herald, Hundreds may have been exposed to measles.

125 Ibid.

${ }^{126}$ WHO factsheet, measles.

${ }^{127}$ New Zealand Herald, Hundreds may have been exposed to measles.

${ }^{128}$ WHO factsheet, measles, pg. 3.
} 
Joshua Mclvor, The Ethics of Infectious Disease Control.

\section{Conclusions.}

We have now demonstrated how to use significant biological features of infectious diseases to come to practical conclusions that uphold important and well founded ethical principles. Furthermore, we have shown that by utilising said biological features to generate an infectious disease taxonomy, we can arrive at pertinent ethical conclusions about various infectious diseases through their key similarities and differences to other infectious diseases. By utilising the infectious disease taxonomy we created here, we have identified the root causes of our ethical conclusions when it comes to infectious disease control: the various biological features of diseases that dramatically alter the way in which they are controlled. Through the taxonomy, we can get at the important ethical considerations for various diseases in a fast, organised, and sensible manner.

What can be said about the conclusions arrived at in this thesis? What is their practical significance? We started out to answer four main questions in the area of infectious disease control and I believe we have succeeded. The questions are listed at the bottom of page three. Let us see how we would respond to them now, knowing what we know. The first question is: when, if ever, is it ethically justified to use experimental treatment? This was the first questions for us to go into detail on and we used the EVD outbreak to examine it. I argued that in the case of the 2014 EVD outbreak, we would be ethically justified in utilising experimental treatment and I defended the point against a few objections. But to properly answer yes to the question we can't simply acknowledge it solely in the context of the EVD outbreak and this was made clear early on. We would need a means by which to answer the question for all infectious disease cases. By utilising the disease taxonomy, we now have that means. We now know that stage three diseases are much more likely to warrant the use of experimental treatment during outbreaks as the opportunity to study the disease in humans often only occurs during outbreaks. Furthermore, diseases that are more easily spread, such as through indirect contact or through the air, are more likely to fulfil the harm principle and thus warrant measures such as the use of experimental treatment. Additionally, if a disease is highly virulent we have the same issue as above: the harm principle is more likely to be met and thus the potential benefits of using experimental treatment (saving lives) is likely to outweigh the costs 
Joshua Mclvor, The Ethics of Infectious Disease Control.

(potential unknown side effects). Stage four and five diseases tend to persist in the human population for long periods of time and thus can be studied year round, thus there is not an imperative to rush the development of treatment due to a small window of opportunity. Therefore, the use of experimental treatment may not always be justified, unless a given outbreak is particularly bad. So we can conclude that the answer to the first question posed in the introduction to this thesis is: it is very likely that the use of experimental treatments during outbreaks of highly virulent, stage three diseases, which are spread through either indirect contact or through the air, is ethically justified. Furthermore, it is likely that the use of experimental treatments during bad outbreaks of highly virulent, stage four and five diseases, which are spread through either indirect contact or through the air, is ethically justified. Both of these answers will of course assume that Upshur's four ethical principles will be met. This is not an exact answer, but that's exactly the point: you cannot account for practical concerns of particular disease outbreaks until they occur. However, using the disease taxonomy table, we are able to quickly come to a probabilistic, ethical conclusion, which immediately gets us pointed in the right direction.

The second question we looked to answer was: when is quarantine/isolation ethically justified? We found that during the 2014 EVD outbreak, quarantine and isolation was ethically justified, although it wasn't carried out well in this particular case. The two biggest biological features that played into our answer to the question of quarantine and isolation were: mode of transmission and virulence. There is little point in quarantining or isolating individuals who don't spread the disease via indirect contact or through the air as there simply isn't reason to believe such measures will dramatically impact the spread of the disease. Furthermore, we found that if a society fulfilled its obligations under the principle of reciprocity, we can conclude that individuals have an obligation to self-isolate, even for diseases with low virulence. So we can say that when it comes to mandatory isolation or quarantine, it is very likely these actions will be ethically justified for highly virulent, stage three, four, and five diseases, which are spread through indirect contact or through the air. Additionally, there is a moral imperative for individuals to self-isolate for diseases with low virulence, assuming society is fulfilling the principle of reciprocity. Finally, the harm principle and least restrictive means principles will mean the measures taken to isolate and 
Joshua Mclvor, The Ethics of Infectious Disease Control.

quarantine individuals, will likely be proportional to the virulence of the disease: the higher the virulence, the more extreme measures need to be taken.

Our answers to questions three and four are interlocked. The questions were: What are the key ethical principles to adhere to when it comes to infectious disease control; and, does our application of ethical principles vary between diseases? We found that the key ethical principles to adhere to when considering infectious disease control measures are: the principles of reciprocity, harm, proportionality, and transparency. When all of these principles are upheld, a particular infectious disease control action can be considered ethically justified. Furthermore, how we apply these principles is going to differ greatly between diseases due to key biological factors. It was ultimately this line of thinking that led to our disease taxonomy table.

I would like to take a moment to comment on the methodology of this thesis. This thesis started with a real-world case study: the 2014 EVD outbreak. By examining the ethical questions that arose during the outbreak, we developed a good sense for the kinds of issues that arise in the ethics of infectious disease control, during real-world scenarios. Furthermore, the case study provided a base from which to ask the question: would we have come to the same conclusions if the disease was different? If we were to come to different conclusions, why would this be the case? This shift in focus allowed us to discuss some of the key ethical principles to be upheld during infectious disease control and highlighted some important differences between our application of these principles to varying diseases. From here, we began to hone in on exactly what the important biological features of diseases are. Ultimately, this led us to the creation of the ethical framework for the infectious disease taxonomy. This framework, I have argued, could be applied to public policy to aid in decision making regarding ethical infectious disease control. It provides a useful methodology for future discussions of the ethics of infectious disease control. So I hope it is clear that I have attempted to guide the reader to our conclusions in a logical manner. Furthermore, I hope these conclusions are easily reachable by more general readers of this thesis, not simply academic philosophers and bioethicists (I touch on this point again in the afterword). Finally, I hope the ethical framework that arises from the infectious disease taxonomy table, can aid in further discussion around the ethics of infectious disease control and infectious disease control policy. 
Joshua Mclvor, The Ethics of Infectious Disease Control.

\section{References:}

ABC News, "Exclusive: TB Patient Asks Forgiveness but Defends Travel." Retrieved from: http://abcnews.go.com/GMA/OnCall/story?id=3231184\&page=1.

CDC, "Insecticide-Treated Bed Nets" 2015. Retrieved from:

http://www.cdc.gov/malaria/malaria_worldwide/reduction/itn.html.

Donovan, Kevin G., "Ebola, epidemics, and ethics - what we have learned." Philosophy Ethics, and Humanities in Medicine Vol. 9(15), 2014. DOI: 10.1186/1747-5341-9-15.

Edwards, Sarah J. L., "Experimental Treatments for Ebola." Research Ethics Vol. 10(3), 2014: 126-128. DOI: 10.1177/1747016114551121.

Fidler, David P.; Gostin, Lawrence O.; and Howard, Markel, "Through the Quarantine Looking Glass: Drug-Resistant Tuberculosis and Public Health Governance, Law, and Ethics." Faculty Publications Paper 371, 2007: 616-628. Retrievable from: http://www.repository.law.indiana.edu/facpub/371.

Goodman, Jesse L., "Studying 'Secret Serums' - Toward Safe, Effective Ebola Treatments." New England Journal of Medicine 371, 2014: 1086-1089. DOI: 10.1056/NEJMp1409817.

Gostin, Lawrence O.; Friedman, Eric; and Hougendobler, Dan, "West Africa's Ebola Epidemic is Out of Control, but Never Had to Happen." O'Neill Institute, briefing paper no. 9, 2014.

Guardian, The, "Pfizer pay out to Nigerian families of meningitis drug trial victims." Retrieved from: http://www.theguardian.com/world/2011/aug/11/pfizer-nigeriameningitis-drug-compensation.

Gupta, S. and Dellorto, D. "Experimental drug likely saved Ebola patients." CNN, 2014. Article retrieved from: http://edition.cnn.com/2014/08/04/health/experimentalebola-serum/.

Harris, John and Holm, Soren, "Is There A Moral Obligation Not To Infect Others?" British Medical Journal, Vol. 311(7014), 1995: 1215-1217. 
Joshua Mclvor, The Ethics of Infectious Disease Control.

Ministry of Health, "New Zealand Influenza Pandemic Plan: A framework for action." Wellington: Ministry of Health, 2010. Retrieved from: http://www.health.govt.nz/system/files/documents/publications/nzipapframework-for-action-apr2010.pdf.

National Ethics Advisory Committee, "Getting Through Together: Ethical values for a pandemic." Wellington: Ministry of Health, 2007. Retrieved from: http://neac.health.govt.nz/system/files/documents/publications/getting-throughtogether-jul07.pdf.

Nelson, Kenrad E., Chapter Two of "Infectious Disease Epidemiology $-3^{\text {rd }}$ Ed." Jones \& Bartlett Publishers, Burlington MA, 2014: 19-44. ISBN: 978-1-4496-8379-5.

New Zealand Herald, "Hundreds may have been exposed to measles." 2016. Retrieved from: http://www.nzherald.co.nz/nz/news/article.cfm?c_id=1\&objectid=11591266.

New Zealand Legislation:

- $\quad$ Bill of Rights Act 1990:

http://www.legislation.govt.nz/act/public/1990/0109/latest/whole.html.

- Epidemic Preparedness Act 2006:

http://www.legislation.govt.nz/act/public/2006/0085/latest/DLM404459.html.

- Health Act 1956:

http://www.legislation.govt.nz/act/public/1956/0065/latest/DLM305840.html.

- Health (Infectious and Notifiable Diseases) Regulations 1966:

http://www.legislation.govt.nz/regulation/public/1966/0087/latest/DLM24207.html.

- Health (Quarantine) Regulations 1983:

http://legislation.govt.nz/regulation/public/1983/0052/latest/whole.html.

Omonzejele, Peter F., "Ethical Challenges Posed by the Ebola Virus Epidemic in West Africa." Journal of Bioethical Inquiry Vol. 11, 2014: 417-420. DOI: 10.1007/s11673-014-95873. 
Joshua Mclvor, The Ethics of Infectious Disease Control.

Pfizer, "Trovan, Kano State Civil Case - Statement of Defence". Retrieved from: http://www.pfizer.com/sites/default/files/news/trovan_statement_defense_summa ry.pdf.

Quammen, David, "Ebola: The Natural and Human History of a Deadly Virus." WW Norton Publishers, 2014. ISBN: 978-0-393-35155-2.

Sifferlin, Alexandra, "4 Diseases Making a Comeback Thanks to Anti-Vaxxers." TIME, 2014. Retrieved from: http://time.com/27308/4-diseases-making-a-comeback-thanks-toanti-vaxxers/.

Tracey, Elizabeth, "ZMapp and Ebola” John Hopkins Medicine: Medical News Commentary, 2014. Retrieved from:

http://podblog.blogs.hopkinsmedicine.org/2014/09/06/zmapp-and-ebola/.

Upshur, Ross, "The Ethics of Quarantine." Virtual Mentor Vol. 5(11), 2003.

Upshur, Ross, "Ethics and Infectious Disease." Bulletin of the World Health Organization, Vol. 86(8), 2008. Retrieved from http://www.who.int/bulletin/volumes/86/8/08056242/en/.

West's Encyclopaedia of American Law, "Food and Drug Administration" The Gale Group, 2005. Retrieved from:

http://www.encyclopedia.com/topic/Food_and_Drug_Administration.aspx

Wolfe, Nathan D.; Dunavan, Claire Panosian; and Diamond, Jared, "Origins of major human infectious diseases." Nature vol. 477, 2007: 279-283. DOI: 10.1038/nature05775. Supplementary notes retrievable from: http://www.nature.com/nature/journal/v447/n7142/suppinfo/nature05775.html.

World Health Organisation, "Ethical considerations for use of unregistered interventions for Ebola viral disease: Report of an advisory panel to WHO." 2014. Retrieved from: http://www.who.int/csr/resources/publications/ebola/ethical-considerations/en/. WHO reference number: WHO/HIS/KER/GHE/14.1. 
Joshua Mclvor, The Ethics of Infectious Disease Control.

World Health Organisation, "Ebola response: What needs to happen in 2015" 2015. Retrieved from: http://www.who.int/csr/disease/ebola/one-year-report/responsein-2015/en/.

World Health Organisation, "Health worker Ebola infections in Guinea, Liberia and Sierra Leone." 2015. Retrieved from:

http://www.who.int/hrh/documents/21may2015_web_final.pdf. WHO reference number: WHO/EVD/SDS/REPORT/2015.1

World Health Organisation, "World on the verge of an effective Ebola vaccine" 2015. Retrieved from: http://www.who.int/mediacentre/news/releases/2015/effectiveebola-vaccine/en/

World Health Organisation, "Factsheets." Factsheets on the various diseases referenced available from: http://www.who.int/topics/infectious_diseases/factsheets/en/. 
Joshua Mclvor, The Ethics of Infectious Disease Control.

\section{See Also:}

Bryan, Charles S.; Call, Theresa J.; and Elliott, Kevin C., "The Ethics of Infection Control: Philosophical Frameworks." Infection Control and Hospital Epidemiology Vol. 28(9), 2007: 1077-1084.

Edwards, Sarah J. L., "The role, remit and function of the research ethics committee -3 . Balancing potential social benefits against risks to subjects." Research Ethics Review Vol. 6(3), 2010: 96-100.

Selgelid, Michael J., "Ethics, Tuberculosis and Globalization." Public Health Ethics Vol. 1(1), 2008: 10-20. DOI: 10.1093/phe/phn001.

Smith, Charles B.; Battin, Margaret P.; Jacobson, Jay A.; Francis, Leslie P.; Botkin, Jeffrey R.; Asplund, Emily P.; Domek, Gretchen J.; and Hawkins, Beverly, "Are there characteristics of infectious diseases that raise special ethical issues?" Developing World Bioethics Vol. 4(1), 2004. ISSN 1471-8847.

Verweij, Marcel, “Obligatory Precautions Against Infection.” Bioethics Vol. 19(4), 2005. ISSN 1467-8519. 
Joshua Mclvor, The Ethics of Infectious Disease Control.

\section{Acknowledgements:}

I would to thank my parents for their constant reminders to do some work on my Master's and my friends who were there to drink a beer with me when I inevitably ignored my parents. My supervisor, Nicholas Agar, deserves thanks for all the feedback and advice, as well as a great deal of patience when I chose to stubbornly stick to my guns on some issues. Additionally, I would be entirely incapable of writing a Master's thesis if it weren't for the Philosophy department at Victoria University. Finally, I'd like to thank Henry Galvin, a PhD microbiology student at Otago University. Henry put a copious amount of time into answering my abundant questions about how diseases work, while walking around the Otago University campus, coffee in hand, in the middle of winter. Had it not been for these long discussions, I am not sure if I could have created my taxonomy or kept my sanity. 
Joshua Mclvor, The Ethics of Infectious Disease Control.

\section{Afterword:}

To the best of my ability within the framework of academia, I have attempted to make this thesis both interesting and comprehensible to the general reader, not just academic philosophers. This unfortunately seems to run counter to the current state of academic philosophy in many parts of the world. I do not have strong opinions on the matter and have nothing but gratitude for those academic philosophers that made my studies interesting, challenging, and enlightening. I do however think that the matter of philosophy students perhaps being encouraged to talk about the abstract and the abstruse over the interesting issues of the real world is worth noting.

An article recently came out on the website "Quartz", quoting philosopher Daniel Dennett's thoughts on the issue. Dennett was quoted as remarking "A great deal of philosophy doesn't really deserve much of a place of the world,"... "Philosophy in some quarters has become self-indulgent, clever play in a vacuum that's not dealing of problems of any intrinsic interest." ${ }^{129}$ The article continues: "This 'cottage industry' certainly isn't helped by the pressure on young philosophy students to publish papers. 'It can take years of hard work to develop the combination of scholarly mastery and technical acumen to work on big, important issues with a long history of philosophical attention,' says Dennett. 'In the meantime, young philosophers are under great pressure to publish, so they find toy topics that they can knock off a clever comment/rebuttal/revival of.'"'130 This is clearly going to vary from university to university, in which respect I was pretty fortunate, but I do believe it to be a strong theme in academic philosophy. I will finish this section with a quote from Sam Harris' book "The Moral Landscape", which is a great quote that relates to this issue:

"Many of my critics fault me for not engaging more directly with the academic literature on moral philosophy. There are two reasons why I haven't done this: First, while I have read a fair amount of this literature, I did not arrive at my position on the relationship between human values and the rest of human knowledge by reading the work of moral philosophers; I came to it by considering the logical implications of our making continued progress in the

\footnotetext{
${ }^{129}$ Quartz One of the most famous living philosophers says much of philosophy today is "self-indulgent". Retrievable from: http://qz.com/768450/one-of-the-most-famous-living-philosophers-says-much-ofphilosophy-today-is-self-indulgent/

${ }^{130}$ Ibid.
} 
Joshua Mclvor, The Ethics of Infectious Disease Control.

sciences of mind. Second, I am convinced that every appearance of terms like "metaethics," "deontology," "noncognitivism," "antirealism," "emotivism," etc., directly increases the amount of boredom in the universe. My goal, both in speaking at conferences like TED and in writing this book, is to start a conversation that a wider audience can engage with and find helpful. Few things would make this goal harder to achieve than for me to speak and write like an academic philosopher. Of course, some discussion of philosophy will be unavoidable, but my approach is to generally make an end run around many of the views and conceptual distinctions that make academic discussions of human values so inaccessible. While this is guaranteed to annoy a few people, the professional philosophers I've consulted seem to understand and support what I am doing."131

${ }^{131}$ Sam Harris, The Moral Landscape: How Science Can Determine Human Values. Notes: Chapter 1, note 1: pg. 252. 